\title{
环糊精相关的超分子自组装最新进展
}

\author{
周冬香 ${ }^{a}$ 孙 涛 ${ }^{a}$ 邓 维 $*, b$ \\ $\left({ }^{a}\right.$ 上海海洋大学食品学院 201306) \\ $\left({ }^{b}\right.$ 上海大学材料学院 纳米科学与技术研究中心 上海 200444)
}

\begin{abstract}
摘要 对基于环糊精的超分子自组装的最新研究进展作了综述. 详细介绍了环糊精为轮、高分子为轴的聚轮烷的制备 及其修饰的方法, 同时还介绍了无高分子参与的环糊精的超分子自组装高分子化合物的制备. 并且对这些超分子在智 能材料、生物医药和聚合催化等方面的应用进行了介绍.

关键词 环糊精; 超分子; 聚轮烷; 聚合物; 自组装
\end{abstract}

\section{Recent Progress in Supramolecular Self-Assembly Based on Cyclodextrin}

\author{
Zhou, Dongxiang $^{a} \quad$ Sun, Tao ${ }^{a} \quad$ Deng, Wei ${ }^{*}, b$ \\ ( ${ }^{a}$ College of Food Science \& Technology, Shanghai Ocean University, Shanghai 20090) \\ ( ${ }^{b}$ Nano-Science \& Technology Research Center, Shanghai University, Shanghai 200444)
}

\begin{abstract}
The supramolecular material has been widely used as a type of drug/gene delivery, molecule device, molecule sensor and catalysis. Due to their excellent biologic and optical properties, cyclodextrins have recently attracted increasingly more attention. In this review the newest development of the supramolecular self-assembly based on cyclodextrin is summarized. The article provided detail information about the synthesis and applications of both of rotaxanes formed by cyclodextrin ring and polymer axes, and supramolecular polymer without polymer backbone.
\end{abstract}

Keywords cyclodextrin; supramolecule; rotaxane; polymer; self-assembly

超分子结构广泛存在于生物体中, 对于生命体的功 能起着重要的作用, 超分子自组装化学受到了越来越多 的化学家都关注和研究. Lehn 首次提出了超分子的概 念——“超越分子的多个分子”, Cram, Pedersen 和 Lehn 对于超分子深入广泛研究, 他们的开创性工作共同荣获 了 1987 年的诺贝尔奖. 超分子是多个分子间通过非键 合作用聚集在一起的分子集合体，结合力一般有氢键， $\pi-\pi$ 共轭和亲水疏水作用等, 具有刺激一反应的性能, 因 而被广泛的应用在设计合成智能材料、药物/基因载体、 分子器件和分子传感器等方面. 同时超分子材料可以有 效地模拟生物体的功能, 对于分子选择、手性催化、电 子转移以及离子通道等都有着深入的研究应用.

多种分子可以构建超分子体系, 比如冠醚, 环糊精, 葫芦脲和环联吡啶等, 其中环糊精由于它的无毒、生物
降解、对光无吸收等性能而受到广泛的关注, 越来越多 地被应用在生物、光学和传感器等方面. 环糊精是一种 来自于淀粉的环状材料, 其结构是葡萄糖单体通过 $1,4 \alpha$ 连接的环状分子. 在水相中, 通过分子内氢键作用形成 稳定的桶状结构, 外围是亲水性表层而易溶于水溶液 中, 内部是疏水性的空腔, 可以有效地包含疏水性的小 分子，而形成主客体作用(环糊精称为主体，包含的小 分子称为客体，这种通过疏水性作用的结合成为主客体 作用 $)^{[1]}$. 常见的环糊精有 $6,7,8$ 个葡萄糖单体构成, 分 别称为 $\alpha, \beta$ 和 $\gamma$-环糊精. 三种环糊精的内腔高度为 0.79 $\mathrm{nm}$, 直径分别为 $0.47,0.60$ 和 $0.75 \mathrm{~nm}$, 不同的分子直径 对于分子的直径有着特定的选择性，因而能在分子识别 和立体化学中有着广泛的应用.

\footnotetext{
*E-mail:wdeng@shu.edu.cn

Received June 8, 2011; revised August 29, 2011; accepted October 14, 2011. of Hunan Education (No. 10c0268).

上海市东方学者、上海市教育委员会重点学科建设(No. J50704)、湖南教育厅自然科学基金(No. 10c0268)资助项目.
}

Project supported by the Eastern Scholar, the Key Project of Shanghai Municipal Education Commission (No. J50704), and the Natural Sciences Foundation 


\section{1 环糊精轮烷}

轮烷是一个或多个环状分子和一个或多个链状分 子为轴组成的分子集合, 其链分子作轴穿过环分子的空 腔, 两端结合有体积较大分子以防止轴分子的滑出, 从 而形成了稳定的轮烷结构. 环糊精的疏水性作用可以有 效地包合疏水性的小分子和高分子，构建成轮烷或高分 子轮烷. 常用的合成轮烷的方式为, 首先在饱和的环糊 精溶液中加入疏水性的高分子轴, 产生不溶的假轮烷 (Pseudo-Rotaxane)(由于在有机溶剂中仍旧可以抽出高 分子轴, 破坏轮烷结构, 所以称为假轮烷); 然后通过高 效率的反应导入大分子作为轴塞(Stopper), 从而形成了 稳定的环糊精轮烷, 此类合成步骤称为穿入过程 (Threading Procedure). 可以作为轴的高分子材料相当 广泛，具有合适直径的疏水性线形分子都可以应用为轮 烷的轴，其包括聚醚、聚酯、聚硅烷和聚硅醚等(表1). 当 然由此类分子为基元的星形和侧链型的高分子也可以 有效地应用为轴分子材料. 不同环糊精的孔径的不同, 且内腔的疏水性能也有所不同，因而对不同的分子有着 一定的选择性. 比如高分子 PEG, 分子直径小且具有一 定的亲水性，和 $\alpha$ - CD 反应制备轮烷产率高达 $90 \%$ 以上， 却和 $\beta-\mathrm{CD}$ 及 $\gamma-\mathrm{CD}$ 几乎得不到产品. 但是对于直径较大 且疏水性很强的 PPG, $\beta-\mathrm{CD}$ 和 $\gamma-\mathrm{CD}$ 都得到了很好的结 果, $\alpha-\mathrm{CD}$ 却完全得不到产品.

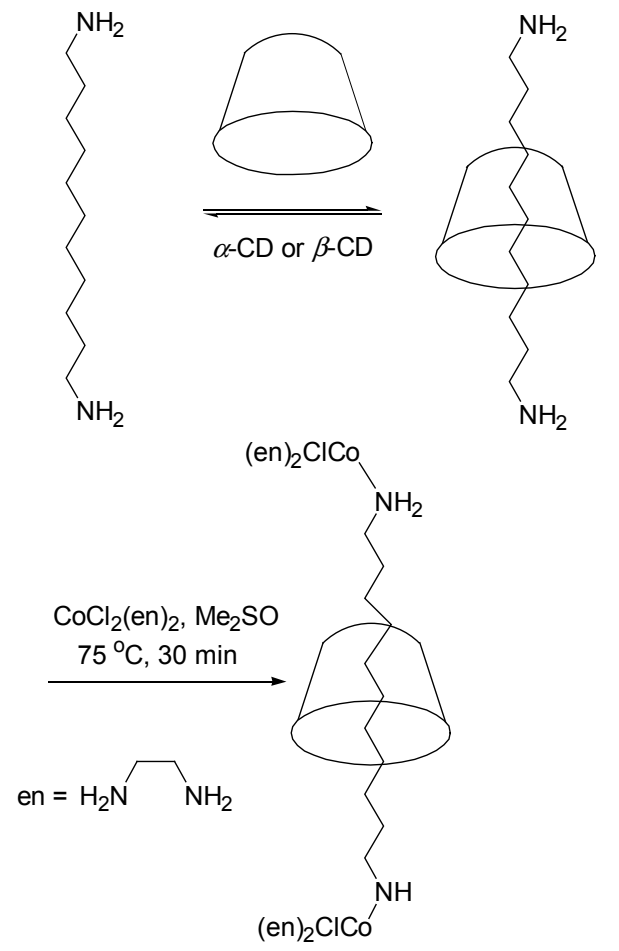

Scheme 1

轮烷的制备研究已近 30 年, 科学家们在努力寻找
容易操作，方法简便、高效并且具有功能化的轮烷分子. 在制备轮烷中, 除了轴分子外, 另一个很重要的因素在 轴塞的选择. 合适的轴塞应该可以在水相中高效地和端 基反应，有效地防止轴分子脱离而提高产率. 其次轴塞 分子的空间结构的选择上需要体积足够大并且稳定，这 样才能形成稳定的轮烷结构. 在 1981 年, Ogino ${ }^{[2]}$ 就首次 报道了通过穿入方法以十一烷二胺为轴和钴的络合物 为轴塞的轮烷. 十一烷二胺作为轴穿过 $\alpha-\mathrm{CD}$ 或者 $\beta-\mathrm{CD}$ 的空腔, 然后端位的胺基和钴盐反应形成络合物作为轴 塞分子. 在 DMSO 溶剂中, 当等物质的量的 $\beta-\mathrm{CD}$ 和十 一烷二胺反应后, 2 equiv. 钴盐络合物作为轴塞, 其反应 的最好收率只有 $19 \%$ (Scheme 1). 这是首次合成稳定的 轮烷结构, 为轮烷的研究提供了可能, 但是由于使用的 有机溶剂作为反应溶剂, 对产率和包合率的影响都比较 大.

Krauter 等 ${ }^{[3]}$ 采用 $\mathrm{B}_{12}$ 的钴盐络合物为轴塞更有效地 制备了这种烷基为轴的轮烷. 其首先通过过量的轮烷轴 和 $\mathrm{B}_{12}$ 的钴盐反应，合成了半轴塞型的轴分子; 在穿入 $a-\mathrm{CD}$ 分子后，另一端被相同的钴盐络合物阻塞上，而 形成了稳定轮烷分子 (Scheme 2). 对比轮烷和纯轴分子 的核磁谱图，由于环糊精的化学屏蔽作用，可以清楚地 看到轴分子的化学位移，从而证明了轮烷的分子结构. 该方法使用了预阻一端的方式，并且在水相中反应，有 效地提高了反应产率.

由于烷基轴和环糊精的包合能力比较弱，从而很难 形成不溶于水的假轮烷中间体和最终产物, 因此比较难 于纯化并且产率也相对比较低. 一个有效的改变是使用 芳香性的轴分子替代烷基轴，其和环糊精的包和能力大 为增强, 穿入的环糊精数目很容易达到空间饱和状态, 并且中间态或最终产物一般不溶于水，极大地方便了纯 化的问题并且收率也大为提高。一个例子就是 Lawrence 等 ${ }^{[4]}$ 报道的联苯和甲基化的 $\beta$-环糊精制备的轮 烷: 端基烷胺基修饰的联苯和甲基化 $\beta$-环糊精在水中形 成假中间体轮烷，水相中添加四苯基硼钠其取代原来端 基的氯离子, 形成了不溶于水的四苯基硼盐为轴塞的轮 烷，其结构通过氢核磁和碳核磁均得到证明(Scheme 3). 由于其结构是立体大结构的盐为轴塞的结构, 因此对于 离子交换的试剂具备了一定的反应性. 在三乙胺存在 下，阴离子很容易被脱轴塞化，而导致环糊精的滑出.

卟啉在生物上广泛存在, 对于光合作用起着关键作 用, 因此在和电子转移、能量转移和光催化等方面有着 重要的应用价值. 基于上述相同的结构和机理，Lawrence 等 ${ }^{[5]}$ 同时制备了四苯基卟啉为轴的轮烷分子. 四苯 基修饰的吓啉对甲基化的 $\beta$ - $\mathrm{CD}$ 具有很强的包合能力, 基于空间位置和立体构象的选择，四苯基卟啉为核心， 
表 1 环糊精为轮、高分子为轴的聚轮烷的形成

Table 1 Synthesis of both of rotaxanes formed by cyclodextrin ring and polymer axes

\begin{tabular}{|c|c|c|c|c|c|c|c|c|c|c|c|}
\hline \multirow{2}{*}{ 类型 } & \multicolumn{2}{|c|}{ 高分子 } & \multicolumn{3}{|c|}{ 环糊精构型 } & \multirow{2}{*}{ 类型 } & \multicolumn{2}{|c|}{ 高分子 } & \multicolumn{3}{|c|}{ 环糊精构型 } \\
\hline & 名称 & 结构 & $\alpha$ & $\beta$ & $\gamma$ & & 名称 & 结构 & $\alpha$ & $\beta$ & $\gamma$ \\
\hline & $\mathrm{OE}$ & $\left(\mathrm{C}^{\mathrm{H}}-\mathrm{C}^{\mathrm{H}}\right)_{n}$ & $\bigcirc$ & $x$ & $x$ & & PTMO & 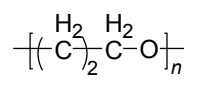 & $\bigcirc$ & $\mathrm{O}$ & $x$ \\
\hline & PP & $\begin{array}{c}\left.\stackrel{\mathrm{I}_{3} \mathrm{H}_{2}}{\mathrm{C}}-\mathrm{C}^{-}\right)_{n} \\
(\mathrm{H})\end{array}$ & $\times$ & $\bigcirc$ & $\bigcirc$ & & PTHF & $\left.\left.\left(\mathrm{C}^{\mathrm{H}}\right)_{3}\right)^{\mathrm{H}_{2}} \mathrm{C}-\mathrm{O}\right)_{n}$ & $\bigcirc$ & $\times$ & $\bigcirc$ \\
\hline & PIB & $\begin{array}{l}\mathrm{CH}_{3 \mathrm{H}_{2}} \\
\left(\begin{array}{l}\mathrm{C} \\
\stackrel{\mathrm{C}}{\mathrm{C}}-\mathrm{C} \\
\mathrm{CH}_{3}\end{array}\right)_{n}\end{array}$ & $x$ & $x$ & $\bigcirc$ & & PMVE & 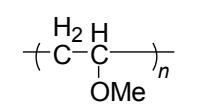 & $x$ & $x$ & $\bigcirc$ \\
\hline
\end{tabular}

聚烯烃

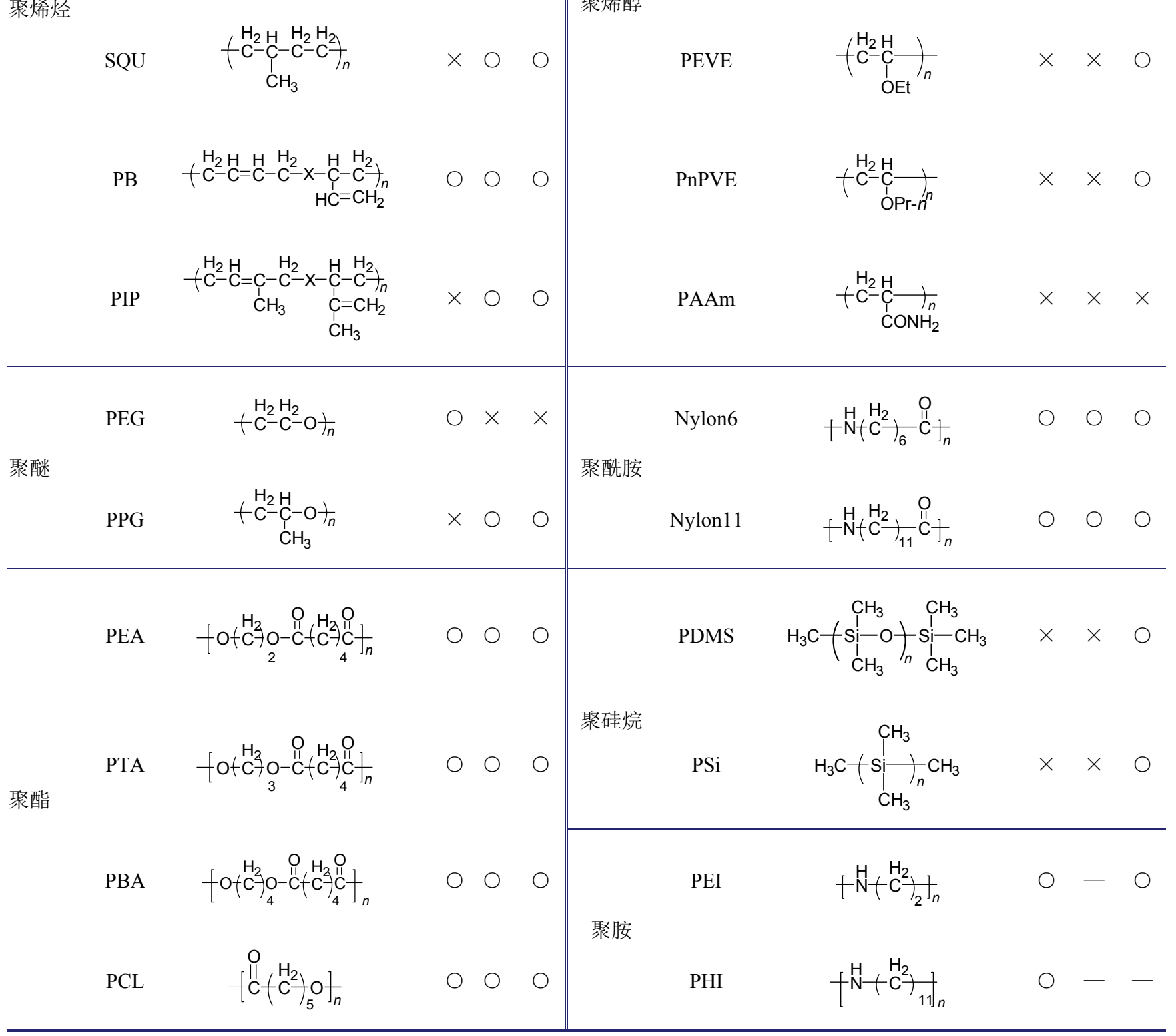

聚烯醇

$\overline{{ }^{a}} \mathrm{O}$ : 可生成轮烷结构; $\times$ : 无轮烷生成; 一: 未见文献报道. 


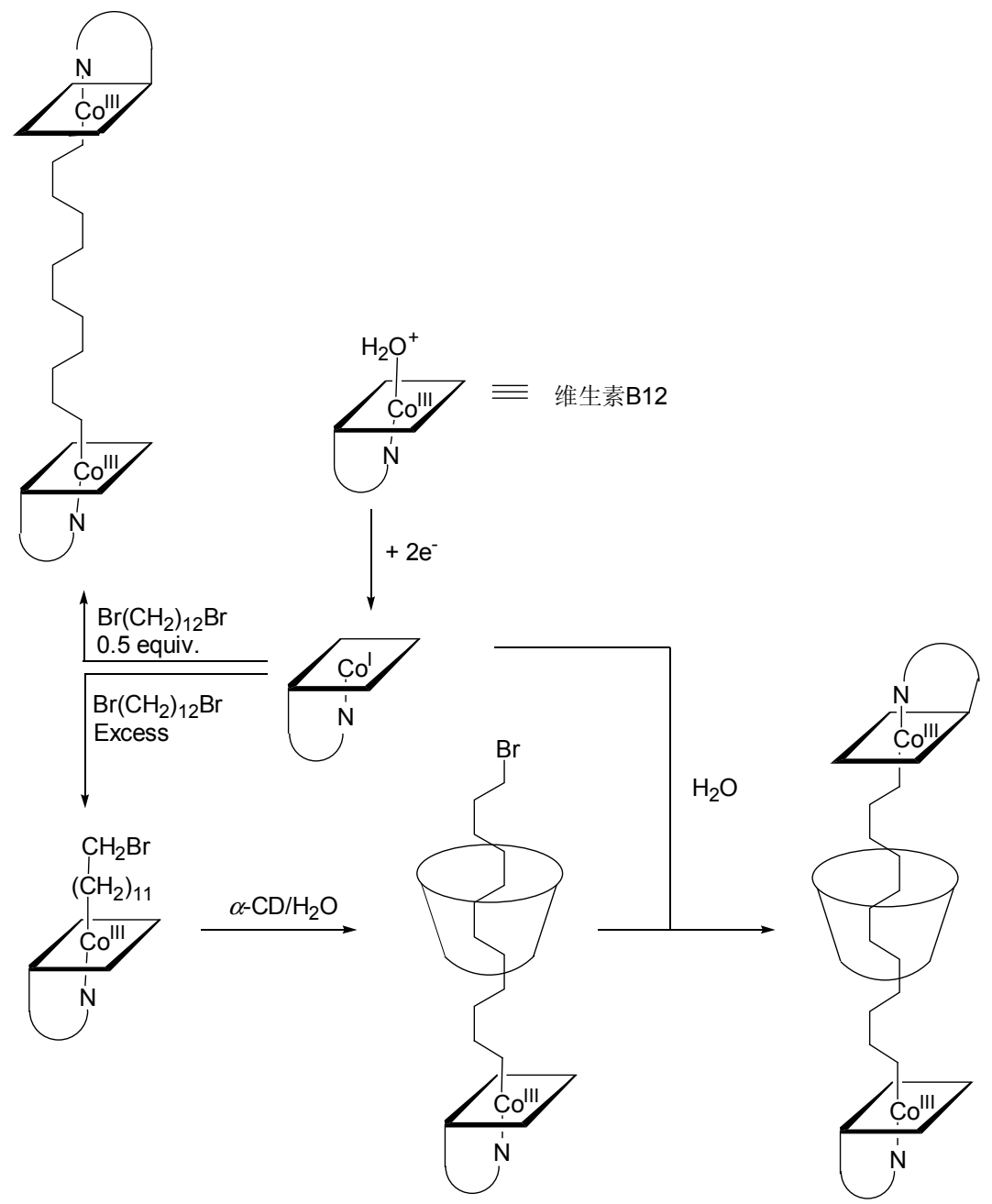

Scheme 2

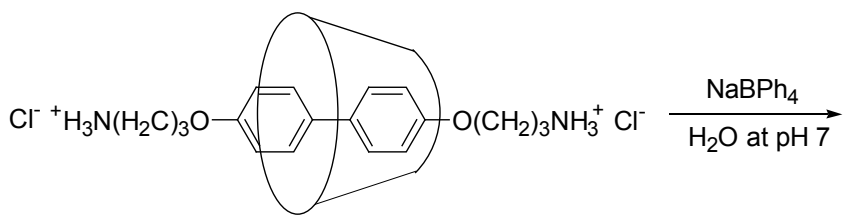

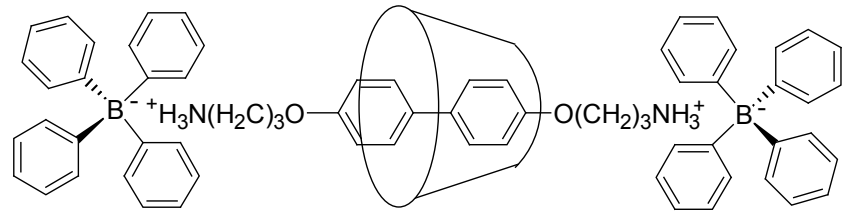

Scheme 3

两物质的量的甲基化 $\beta$-CD 形成稳定的轮烷结构, 端基 以四苯基硼的铵盐为轴塞(Scheme 4). 该轮烷具有一定 的空间结构, 可以有效地研究光合作用模拟和光催化反 应的立体效应，实现天然生物化学的性能模拟.

另一个方法有效地轮烷制备方法是在环糊精的存 在下直接通过小分子的聚合, 该方法不但提供了新的轮
烷制备方法，并且提供了环糊精催化的聚合反应的可 能. 刘育等 ${ }^{[6]}$ 报道了在 $\beta$ - $\mathrm{CD}$ 存在下的联苯二胺和对苯 二醛缩合反应，使用 Sanger 试剂为轴塞，高效地制备了 轮烷分子, 单体分子的转化率接近于 $100 \%$ (Scheme 5). Sanger 试剂因为其分子结构比较小, 不足以完全的阻止 轴链的抽出, 因此在强极性有机溶剂下, 可以将该聚合 

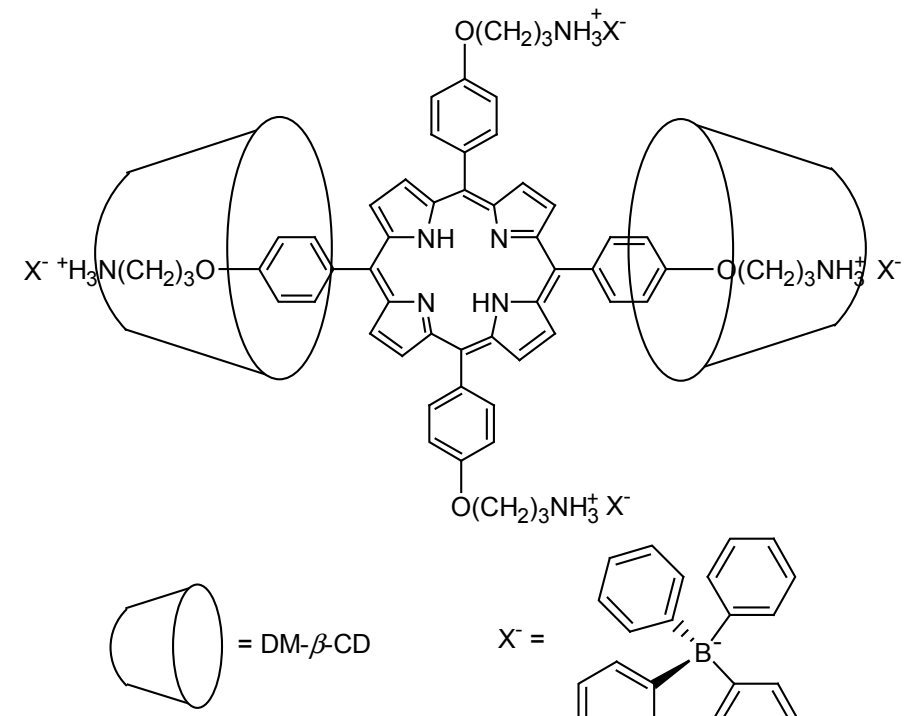

$\mathrm{X}^{-}=$<smiles>c1ccc([B-](c2ccccc2)(c2ccccc2)c2ccccc2)cc1</smiles>

Scheme 4
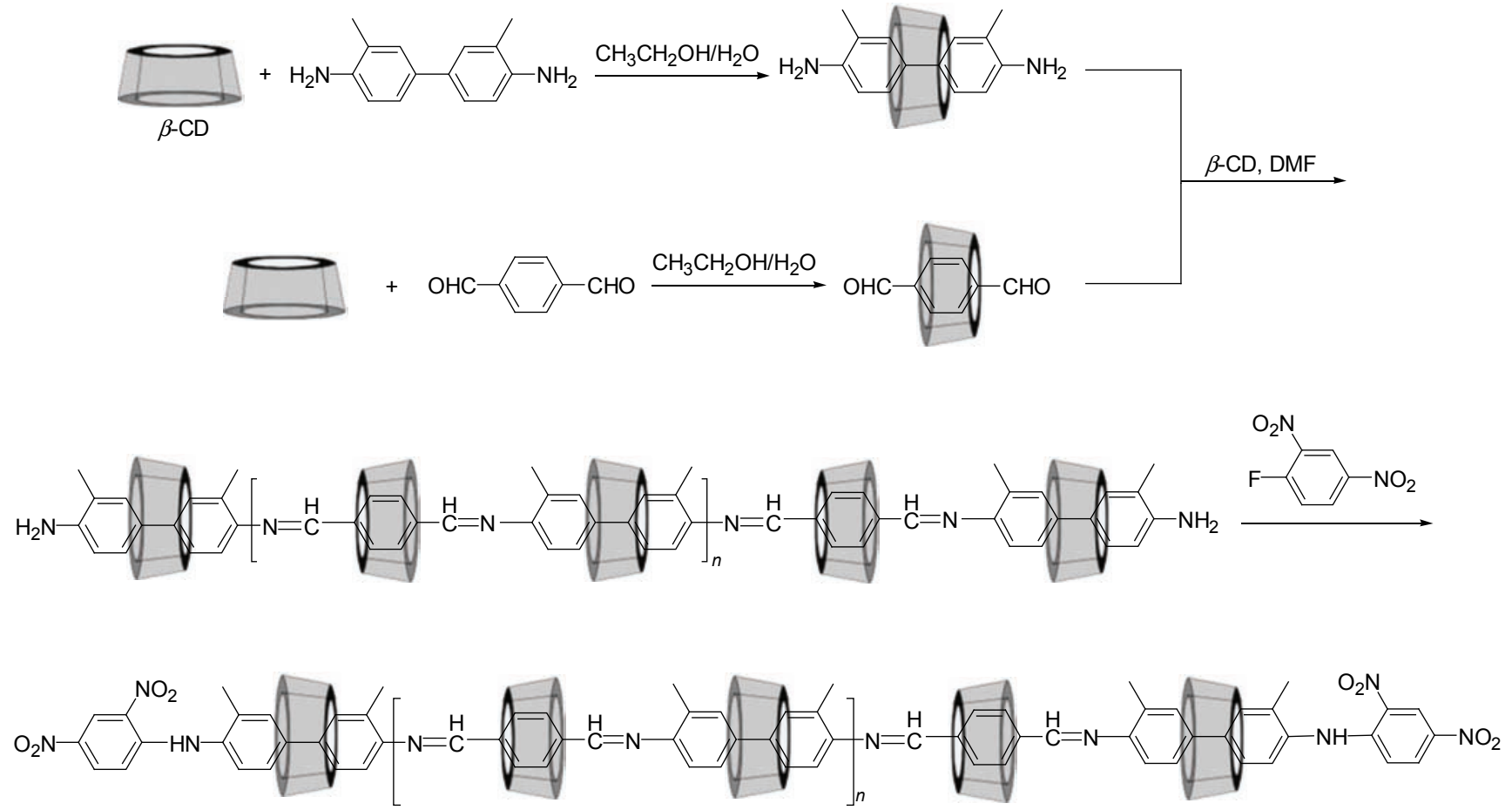

Scheme 5

物分子抽出, 因此是一种伪轮烷的结构. 该分子具有共 轭结构因此可能有一定的导电性能, 对于导电高分子的 设计合成有着一定的价值.

Nepal 等 ${ }^{[7]}$ 报道了对苯二胺和对苯二醛的聚合制备 轮烷, 两者都是很好的客体, 能很好地包合在环糊精内, 通过缩合反应生成 Schiff 碱为连接官能团的聚合物, 其 尾端通过引入足球烯作为轴塞分子而生成能溶解于 DMF 的轮烷分子 (Scheme 6). 足球烯足够大的体积可以
有效地防止高分子轴的脱出而形成了稳定的轮烷结构, 并且足球烯有特殊的电子效应, 在光电化学和材料化学 中提供了潜在的应用价值.

环糊精轮烷一般由轴分子穿入环糊精而成, 因此不 同空间构型的轴分子可以制备具有立体结构的轮烷 (Scheme 7). 对于嵌段共聚物, 环糊精选择性地和部分 链段具有着包合作用, 因此可以形成像嵌段式的轮烷; 对于星形的轴分子, 每一个侧臂均能和环糊精构建成轮 
$\mathrm{H}_{2} \mathrm{~N}$

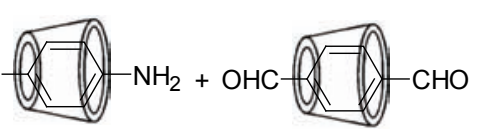<smiles>Cc1ccccc1C(F)(F)F</smiles>

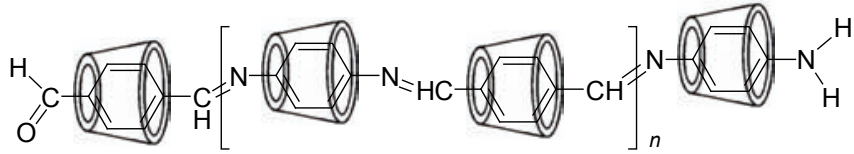

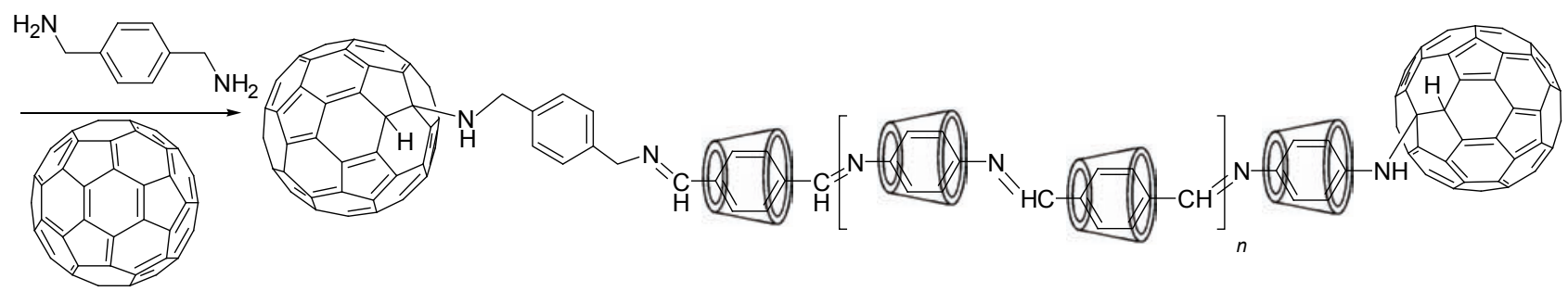

Scheme 6

(a)

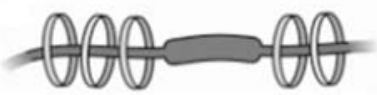

(c)

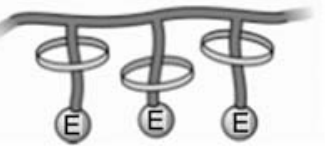

Scheme 7

烷, 而形成了星形结构的轮烷超分子; 另一个重要的轮 烷结构是侧链型轮烷, 轴分子作为侧链接枝在主分子链 上，通过包合作用环糊精分子穿入轴分子，然后封闭而 成. 不同空间结构的高分子对于性能有着重要的影响, 因此具有立体结构的轮烷也受到了广泛关注, 在功能性 材料等方面的具有应用价值.

Ritter 等 ${ }^{[8]}$ 首先报道了侧链轮烷的合成. 其以聚丙 烯酸为主链, 首先预制备了单端封闭化的假轮烷中间 体, 然后氨基和活性的侧链基团反应而得到了新型的侧 链化轮烷结构, 通过类似的合成步骤, Ritter 制备了一系
列的分子结构, 并且进行了广泛得研究(Scheme 8). 同 时, Yamamoto 等 ${ }^{[9]}$ 也合成了相似的侧链的轮烷, 不同的 是其使用的是聚苯并咪唑为主链, 端基三苯甲烷的溴代 烷基链为侧链反应分子的轮烷结构. 其研究了侧链间隔 空间对环糊精穿入率的影响，由于空间效应，当间隔空 间越大的时候，越多的环糊精分子容易被测链轴分子穿 入而形成侧链式轮烷. 这是首次基于早期的轮烷的合成 方法, 得到的侧链型的聚轮烷.

Wei 等 ${ }^{[10]}$ 报道了星形的侧链轮烷, 其以苯乙醇取代 的卟啉为核心, 在辛酸锌的催化下, 以端羟基为起始段, 己内酯开环聚合为聚酯而形成四条臂的星形轴分子，和 $\alpha-C D$ 构成了星形的轮烷结构(Scheme 9). 对比于轴分 子, 轮烷有着更强的苂光型号, 可以应用在分子显像或 者生物探针方面, 更重要的是其在多肽药物载体和释放 等方面有着潜在的应用价值.

一个很有趣的轮烷的构建是利用纳米金作为中心 形成星型轮烷. Kaifer 等 ${ }^{[11]}$ 利用纳米金颗粒为核心制备

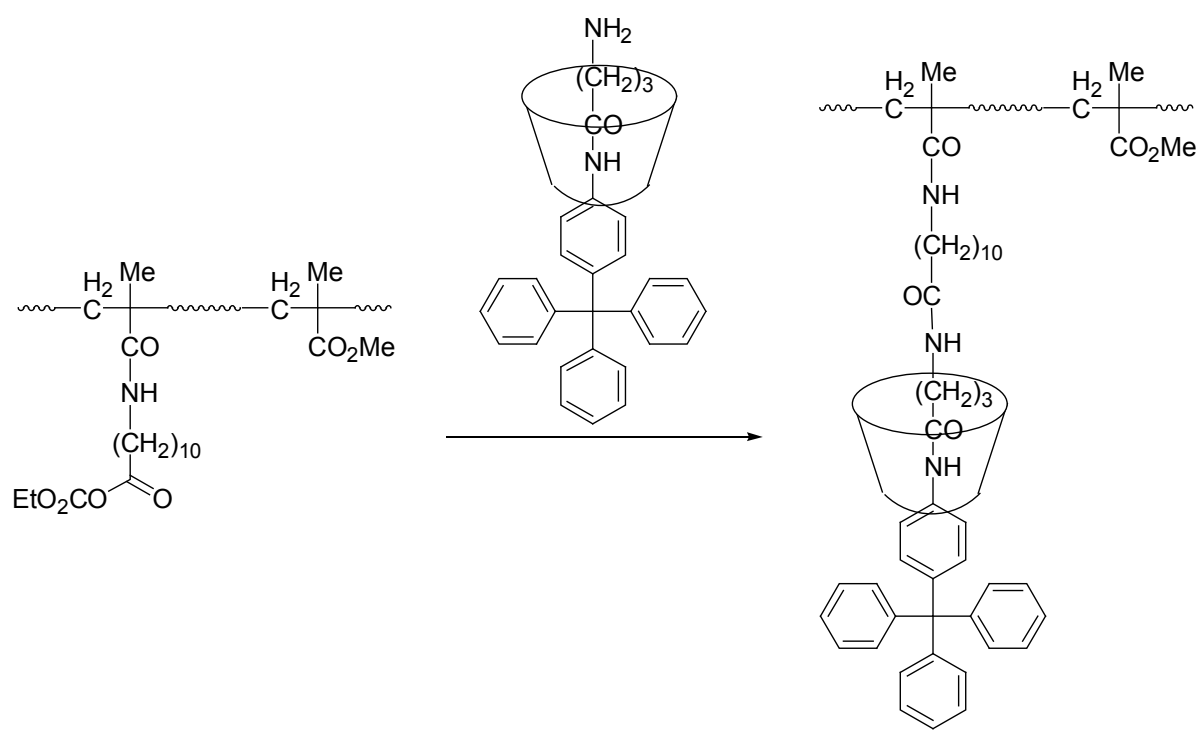

Scheme 8 


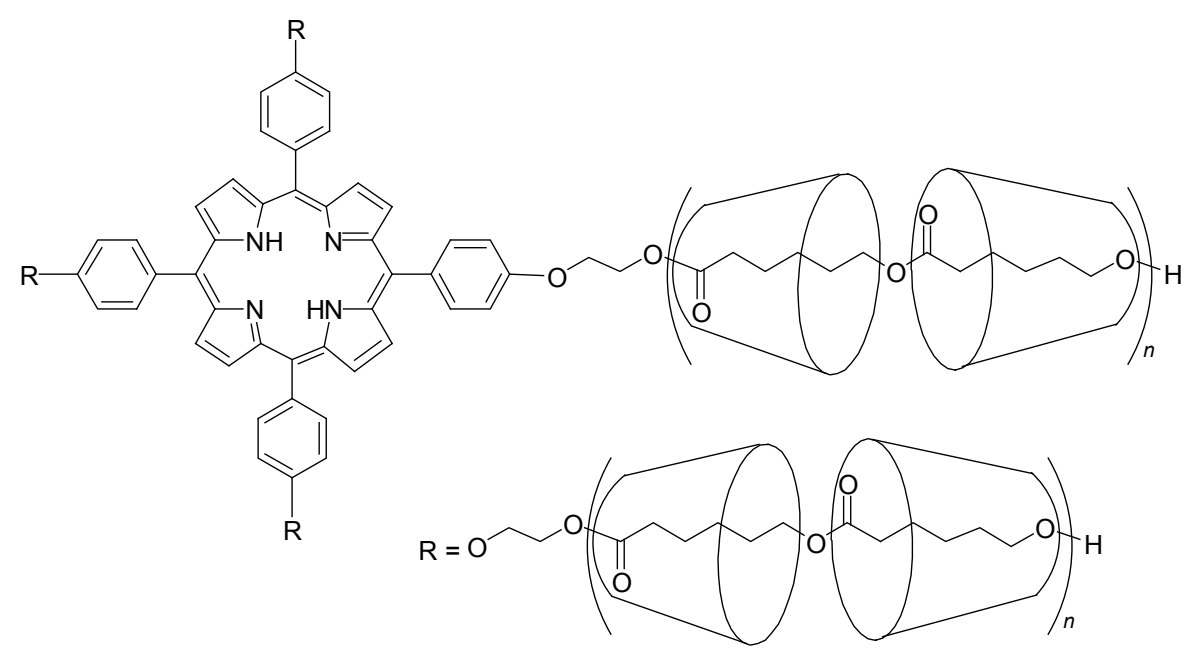

Scheme 9

了星形的轮烷分子, 二茂铁为一端基的直链的烷烃和 $\alpha-\mathrm{CD}$ 形成半轮烷, 然后另一端的硫醇和纳米金表面缩 合而形成稳定的纳米金星形轮烷 (Scheme 10). 纳米金 在生物医药和材料化学上有着广泛的应用, 在生物成 像、药物转移以及光电作用等方面都得到了深入的研究, 对于纳米金的修饰是研究热点, 该方法扩展了纳米金的 功能化修饰.

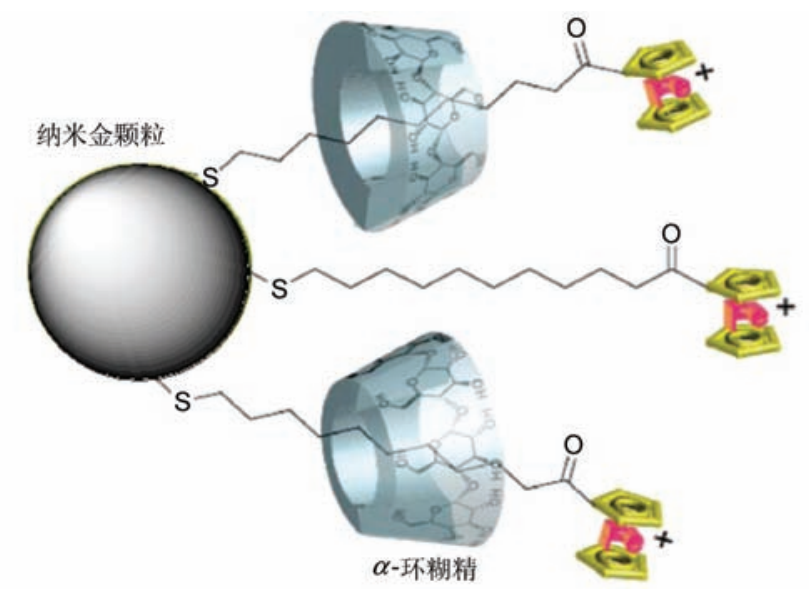

Scheme 10

$\gamma-\mathrm{CD}$ 的分子内腔相对较大, 和一般单链高分子(如 PEG) 结合力过小而难于形成稳定的轮烷结构. 但是 Kamachi 等 ${ }^{[2]}$ 的研究表示, 萘端基封闭的 PEG 分子可以 分子链重叠为双链, 产生足够的空间体积, 从而进入 $\gamma$-CD 内高产率地得到 $\gamma$-CD-PEG 的轮烷分子. 通过苂光 的分析, 发现在轮烷状态的荎分子双分子的荧光, 而在 没有环糊精或者 $\alpha-\mathrm{CD}$ 和 $\beta-\mathrm{CD}$ 的条件下, 只有单分子的 发射苂光被观察到, 从而证明了双链的轮烷结构 (Scheme 11), 这是首次双链聚轮烷结构的报道.

另一个有趣并且有用的轮烷例子是刘育小组 ${ }^{[13]}$ 报 道的双轮烷桥联结构. 首先以氨基-联吡啶机构为连接

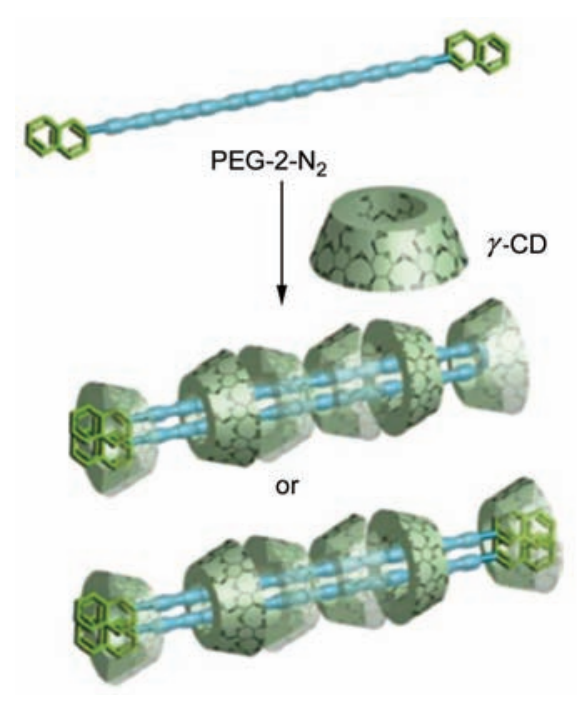

Scheme 11

基团合成了 $\beta$ - $\mathrm{CD}$ 的二聚物, 然后以 $\mathrm{PPG}$ 为轴穿过此二 聚物中心而形成轮烷结构 (Scheme 12). 加入过渡金属 $\mathrm{Cu}^{2+}, \mathrm{Ni}^{2+}$, 氨基和联吡啶的氮原子过渡金属分别形成 四元配合物, 从而形成了双轮烷分子间的规范排列, 通 过透射电镜(TEM)和原子力显微镜(AFM)可以清楚地看 到两条轮烷长链分子紧密地规则地并列排列图像 (Scheme 12). 这种设计有效地组合了超分子轮烷和金 属配位作用，使得轮烷的结构可以有效地利用在金属有 机化学, 从而有更多的光学, 材料学和催化学方面的应 用.

为了简单高效地合成修饰的和不修饰的聚轮烷或 者伪聚轮烷, 化学工作者们也进行了大量的探索. Tardajos 等 ${ }^{[14]}$ 报道了 $\beta$-CD 水溶液在非离子表面活性剂 Igepal CO-520 的存在下, 当水溶液缓慢蒸发干燥后可 以得到三维含有伪轮烷形式的晶体结构. 首先两个环糊 精分子通过头对头的方式, 和表面活性剂形成超分子结 


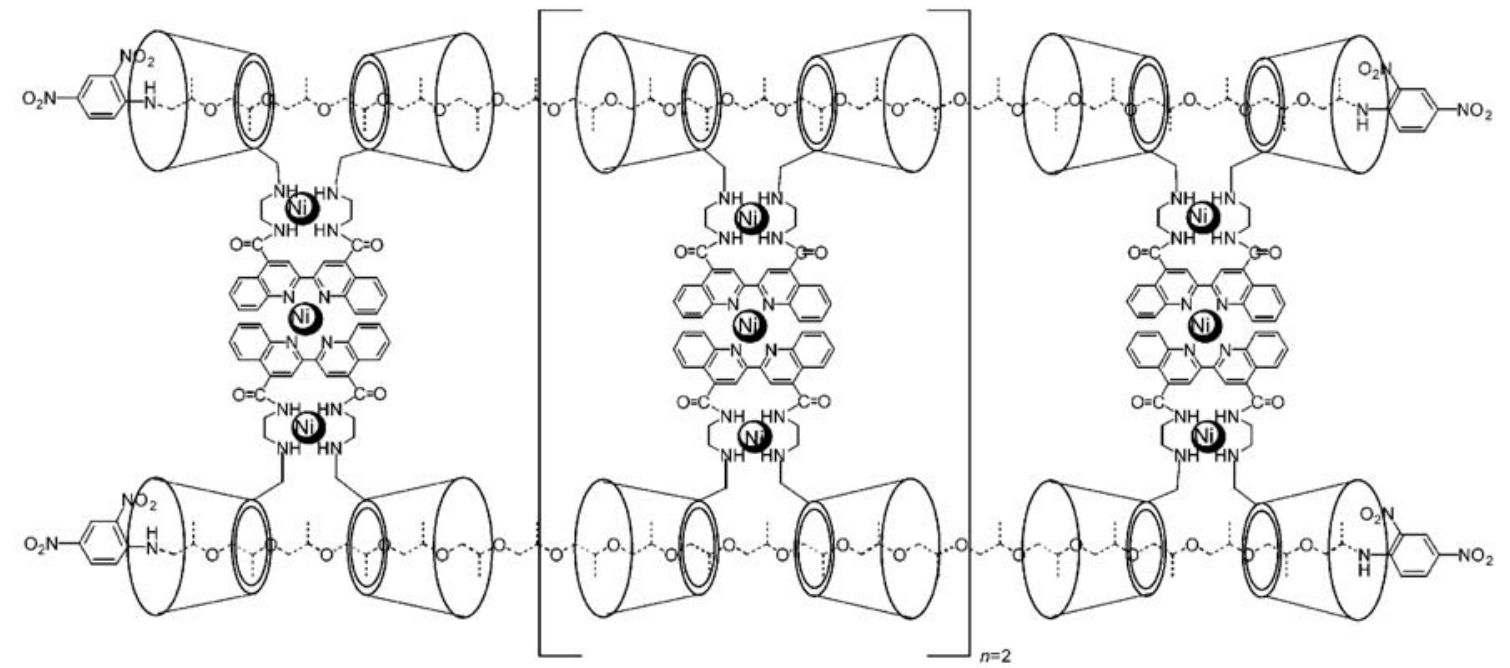

Scheme 12

构, 然后通过环糊精的氢键作用单体叠加而得到了伪轮 烷形式的晶体. 其结果通过 XRD, TEM 和 ${ }^{13} \mathrm{C} \mathrm{CP} / \mathrm{MAS}$ NMR 研究证明了其为层状的晶体结构; 单晶分析其结 构是包含一个 $\beta$ - $\mathrm{CD}$ 二聚体的三斜晶系. 利用单锅合成 法可以有效地简化操作, 减少合成步骤而得到高效地得 到产品, Takata 等 ${ }^{[15]}$ 采用单锅合成法, 在烃类溶剂中使 用多相溶剂系统中, 成功地得到了甲基化的 $\alpha-\mathrm{CD}$ 和聚 四氢呋喃的聚轮烷分子, 其最佳产率达到 $71 \%$. 对不同 烃类溶剂进行优化结果表明异辛烷得到最好的结果, 而 环己烷作溶剂的完全没有聚轮烷结构生成, 且该体系对 于甲基化的 $\beta$-CD 或者非甲基化的 $\alpha-\mathrm{CD}$ 都不适用.

对于环糊精聚轮烷进行修饰是其应用的一个重要 步骤, 一般常用的修饰方法为两步法, 首先制备的聚轮 烷和活化剂反应, 比如 $N, N^{\prime}$-羰基二咪唑、环氧氯丙烷等, 然后和修饰基团连接而成. 最近 Ohkawa 等 ${ }^{[16]}$ 报道了一 种直接引入氨基酸修饰聚轮烷的方式: 首先将氨基酸的 氨基通过叔丁氧甲酰基或苯甲酰保护, 然后和羰基二咪 唑反应活化羒酸基团, 活化的羒酸基团在氯化锂的存在 下和聚轮烷反应, 脱保护后, 得到氨基酸修饰的聚轮烷 (Scheme 13). 这种方式可以有效地避免了通常方式下
活化剂的残留, 氨基酸修饰的毒性低, 可以在生物材料 中得到应用，并且得到的产物的氨基为伯胺，为进一步 修饰提供了可能.

除了先合成聚轮烷再修饰的方式, 将环糊精先修饰 再合成聚轮烷也是一种可行的方式, Auvray 等 ${ }^{\left[{ }^{[17]}\right.}$ 对比了 两种不同的合成方法. 其首先合成了 $\alpha-\mathrm{CD} / \mathrm{PEO}$ 的聚轮 烷, 然后通过高碘酸钠氧化葡萄糖得到二醛结构, 然后 和伯氨基化和物缩合还原得到修饰的聚轮烷. 另一种方 法为 $\alpha-\mathrm{CD}$ 首先通过氧化成醛，和氨基化合物缩合还原 得到修饰的环糊精，修饰的环糊精和 PEO 同样也得到 了修饰聚轮烷结构(Scheme 14). 当然由于溶解度的问 题, 第二种方法得到的产率比较低, 但是可以得到环糊 精修饰均一化的产物.

\section{2 环糊精超分子聚合物}

环糊精形成的疏水性空腔在水相中可以有效地包 含客体小分子而形成分子间的超分子体系. 如果将客体 直接通过化学键和主体环糊精连接时, 这种客体修饰的 环糊精分子在超分子作用下，可以形成分子内和分子间 的超分子体系. 根据进入的方式不同，可以形成三种可
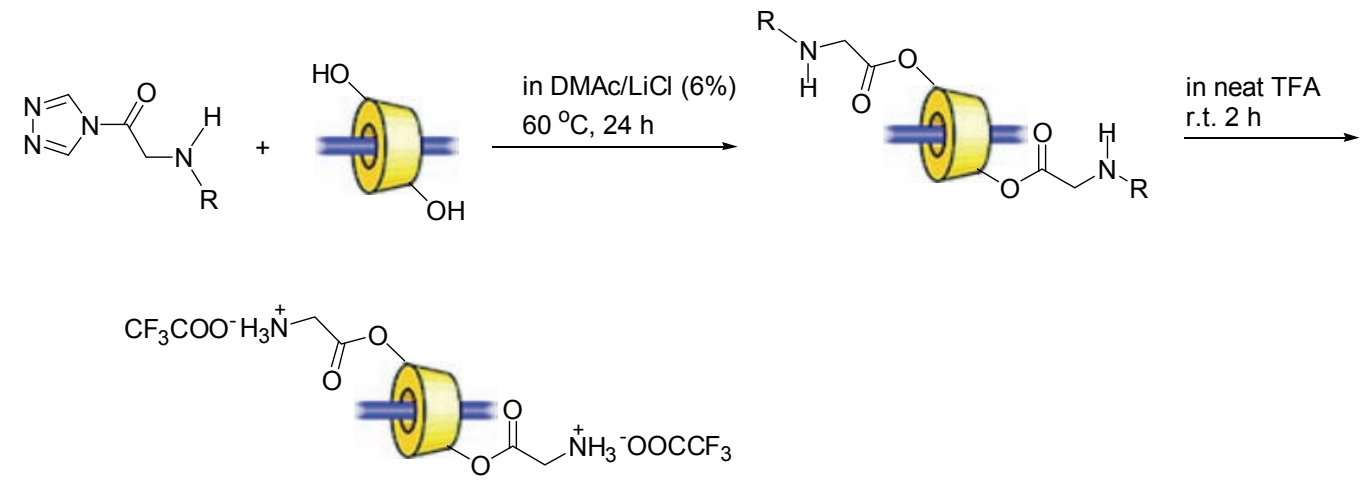

Scheme 13 


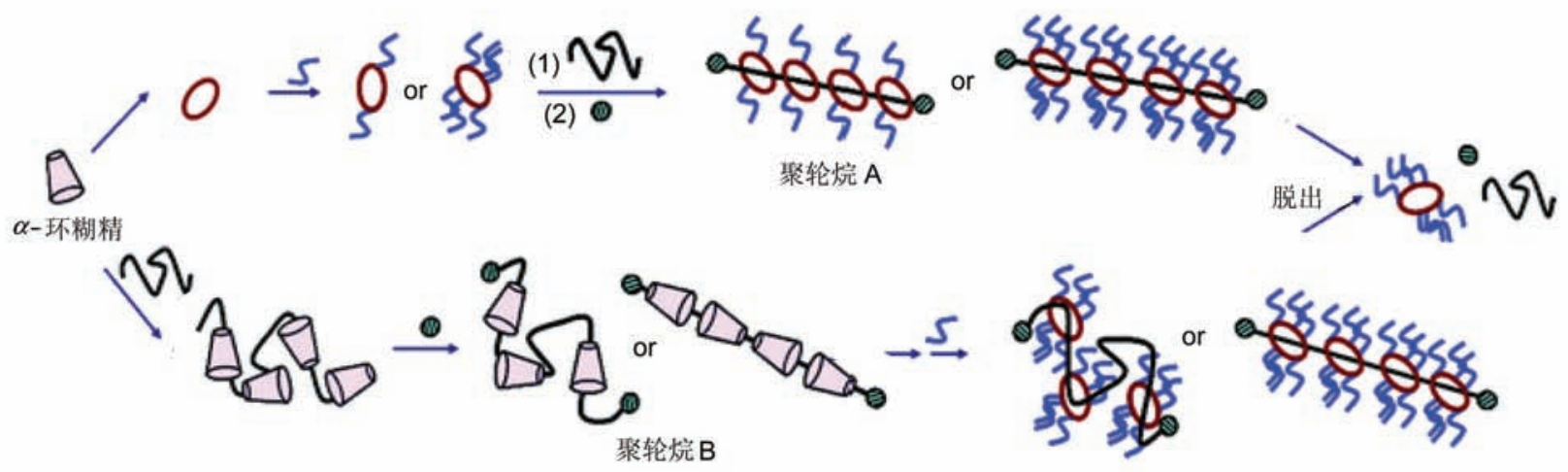

Scheme 14

能的结构: (1)自包合体系——客体分子和环糊精通过柔 软的基团相连接, 连接基团可以弯曲而让客体分子进入 自身环糊精的空腔内，而形成了自包合的单分子结构; (2)超分子二聚物一一当连接基团为刚性的分子时, 自 包合的体系会被阻止，因而只能形成分子间的超分子体 系. 如果单客体修饰的环糊精分子通过头对头或者尾对 尾的方式进行超分子排列, 就可以形成超分子二聚物结 构; (3)超分子聚合物一一刚性的连接的超分子体系中, 当有方向选择性的基团作为客体修饰环糊精分子, 或者 采用特定的方向选择性的结构组合, 可以有效地控制单 客体修饰的环糊精以头对尾的方式进行排列, 而形成超 分子聚合物结构. 自包合和二聚物的超分子体系得到了 广泛的研究, 其性能和单分子有着类似的性质, 对于浓 度和温度等外界因素的相应性比较弱. 对于超分子聚合 物，其整体性能表现的更加具有高分子的特性，同时由 于其通过非共价键的方式构建, 对于一些外在的刺激, 比如热、光、氧化-还原和客体分子等有着对应的反应, 在超分子智能材料方面有着广泛的潜在应用价值, 因而 得到了越来越广泛的研究.

首个超分子聚合物的例子是 6-己二胺修饰的 $\beta-\mathrm{CD}^{[18]}$, 在水中其自身不但是客体分子, 同时也是主体 分子. 在固相状态可以观察到如高分子的结构, 其通过 超分子作用己二胺分子进入相邻的环糊精分子空腔中, 形成线形的规范超分子状态(Scheme 15). Stoddart 等 ${ }^{[19]}$ 称这类型的超分子结构为维菊花链超分子聚合物 (Daisy-chain polymers), 即首尾相扣的结构. 这种类似 于轮烷的构成是轮烷结构扩展, 选择短链的分子替代长 链的高分子轴, 同时轴分子和轮分子以化学键相连. 超 分子聚合物是轮烷分子的扩展, 也打开了超分子自组装 的另一个方向.

Harada 小组 ${ }^{[20]}$ 对超分子聚合物有着广泛的研究, 其合成了不少方式的超分子聚合物结构. 其中一个很好 的例子是 3-对异丁基肉桂酰胺- $\alpha-\mathrm{CD}$, 其可以形成螺旋 状的超分子聚合物. 其圆二色性谱显示, 在极性溶剂没

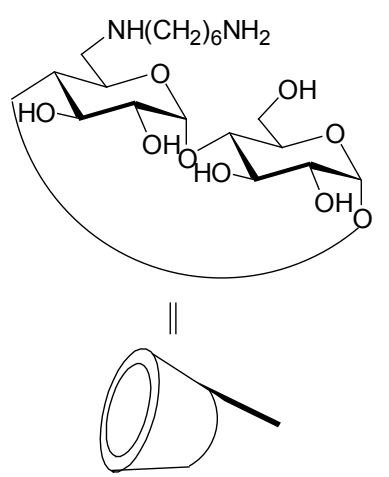

hex- $\beta-C D$

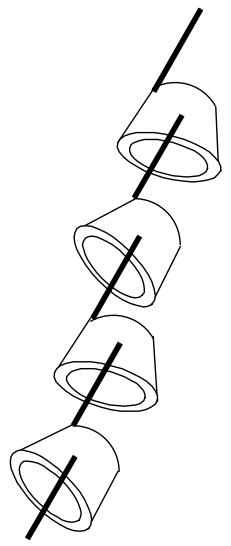

超分子聚合物

\section{Scheme 15}

有偏正光吸收峰, 而在水溶液中, 有正的和负的 Cotton 带吸收峰，其源自于肉桂分子包含于环糊精空腔内而诱 发的圆二色性谱. 对比于聚肉桂类聚合物模型的分析, 其圆二色性谱与左旋高分子结构的谱图相吻合，同时通 过 AFM 可以直接清楚的观测到左旋超分子长链结构 (Scheme 16). 更有趣的是, 客体的位置对于超分子的形 成有着关键的作用，对于 6-对异丁基肉桂酰胺- $\alpha$ - $C D$, 结果表明只有二聚物的生成, 而没有超分子聚合物.

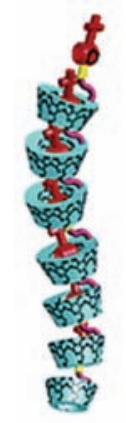

另一个有效的方向选择性的方式是通过空间位阻 效应，可以有效地阻碍二聚物的生成，而向着利于超分 
子聚合物的方向进行. 6-对异丁基肉桂酰胺- $\beta$ - $\mathrm{CD}$ 在水 中形成自包合化合物，但是当金刚烷- $\alpha-\mathrm{CD}$ 加入到 6-对 异丁基肉桂酰胺- $\beta$ - $\mathrm{CD}$ 的水溶液中时, 通过分子间的超 分子作用, 可以形成超分子聚合物. 这是由于金刚烷修 饰的, 其客体体积相对于 $\alpha$-CD 空腔过大, 而位于空腔 外, 但是对于 $\beta-\mathrm{CD}$ 是强客体分子, 可以取代肉桂酰基 团而进入环糊精分子空腔内 ${ }^{[21]}$. 并且由于空间作用, 这 种二聚物无法以头对头的方式继续自组装，而只有向超 分子聚合物的方式聚集(Scheme 17). 这种超分子聚合 物的结构可以通过 AFM 直接观察到线形分子, 同时线 性分子可以被探头通过加力刮蹭而分开，因此也证明了 这种聚合物是通过弱的超分子作用而形成的. 这是首次 通过空间结构控制的超分子的聚合物的合成, 更重要的 是首次利用 AFM 对超分子结合力进行的研究.

当多枝客体修饰分子加入到超分子聚合物体系中 时, 可以有效地提高聚合物的交联作用, 形成网格结构.
Miyawaki 等 ${ }^{[22]}$ 报道了一种超分子网格，当单肉桂酰胺 修饰的 $\alpha-\mathrm{CD}$ 形成的超分子线性聚合物，而多个肉桂酰 胺修饰的 $\alpha-\mathrm{CD}$ 加入时, 其客体分别穿入不同的超分子 链，而产生交联效果形成网格结构，该结构的分子量和 黏度都大大的增加了(Scheme 18). 这种超分子的三维 结构可以作为交联化高分子，有效地拓展了超分子聚合 物的范围。

二元的客体和二元主体分子混合也是一种很好的 超分子高分子制备的方式，其可以通过选择连接分子而 有目的地构建不同的超分子结构. Ohga 等 ${ }^{[23]}$ 合成了多 种不同长度分子连接的二元金刚烷分子，其和苯连接的 二元 $\beta-\mathrm{CD}$ 分子, 对于不同长度的链接基团，可以分别 形成二聚物、线性高聚物和环形的高聚物(Scheme 19). 进一步的研究该体系, Kuad 等 ${ }^{[24]}$ 采用光反应性的二苯 乙烯基团为链接基团取代苯分子，而形成了光刺激反应 性的超分子体系(Scheme 20). 当二苯乙烯为反式结构

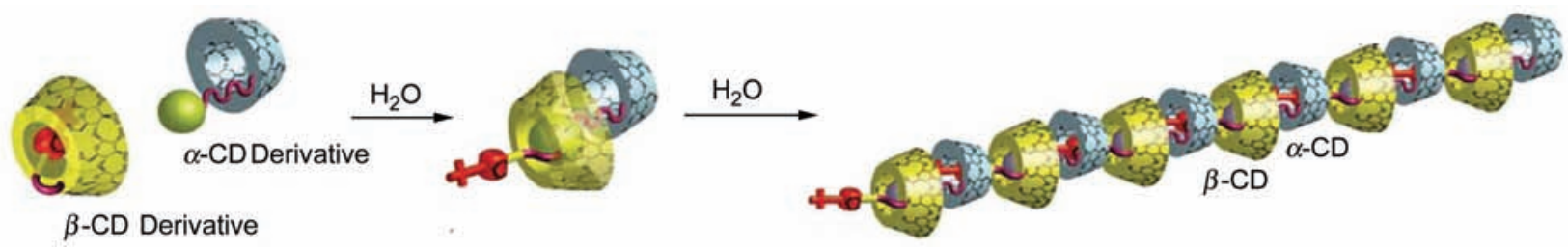

Scheme 17
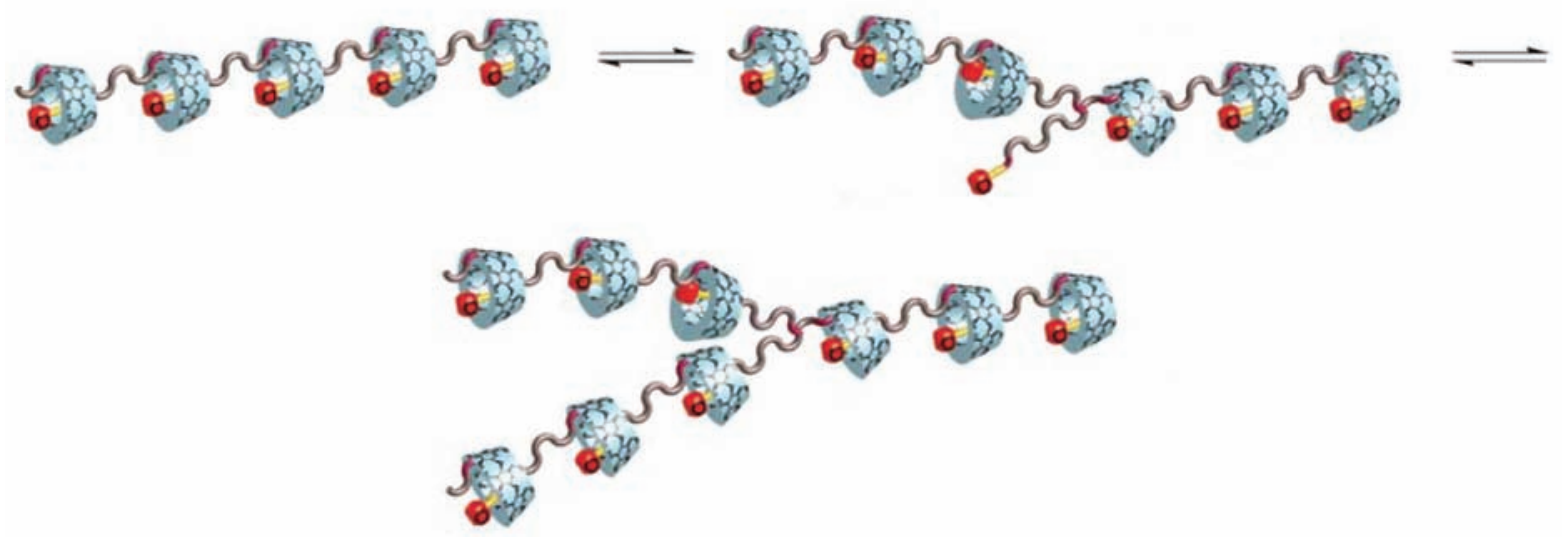

Scheme 18
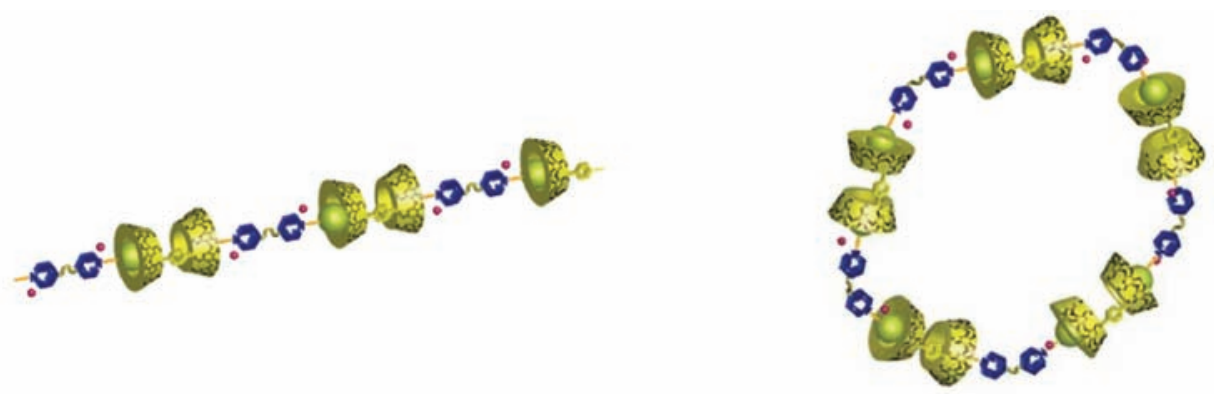

Scheme 19 

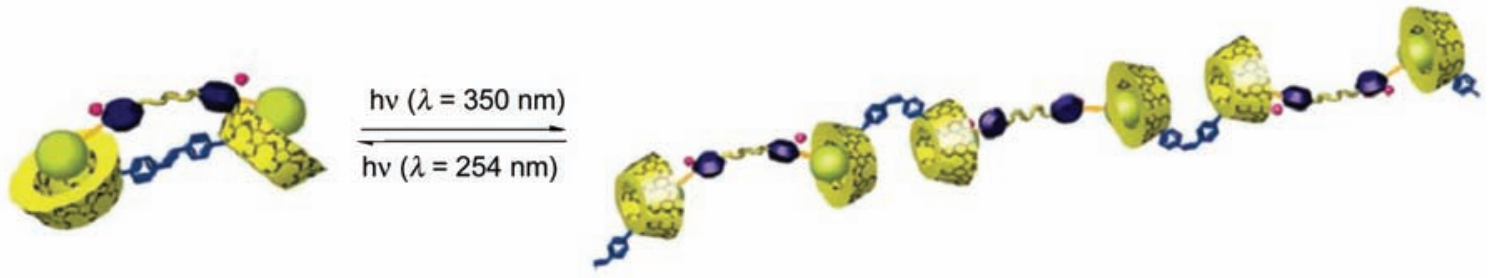

Scheme 20

时, 形成超分子的二聚物, 当反式二苯乙烯在 $35 \mathrm{~nm}$ 的 光照下转化为顺式结构后, 其二聚物结构转化为超分子 的高分子结构, 该高分子结构同样也可以 $254 \mathrm{~nm}$ 的光 照下通过二苯乙烯的光异构化来进行可逆的从高分子 回复到二聚物的结构, 这类光控分子可以应用在信息储 存材料和感应器等方面.

对于更为复杂的二元客体分子 (二元金刚烷)和三元 主体分子(三元环糊精), 其超分子结构表现出了更为复 方法进行了研究, 定性地发现在水溶液中金刚烷二聚物 杂的情况, Tortolini 等 ${ }^{[25]}$ 通过小角度 $\mathrm{X}$ 射线和光散射等 和 $\beta$ 环糊精三聚物可以形成了大环状的、枝权状的和直 链状的超分子聚合物, 通过对其分子动力学的研究发现 其最佳共聚物的分子结构数目为 8 个分子的共聚. 这类 研究为复杂的主客体超分子共聚物提供了理论化的研 究方法.

\section{3 环糊精自组装化合物的应用}

环糊精自组装化合物由于其在性能的独特性, 而在 材料方面得到了广泛的应用研究. 轮烷结构中, 其轴分 子是高分子结构, 因此轮烷具有高分子的性能, 同时由 于每一个环糊精的轮结构是可以自由旋转的, 因此具有 空间的自适配的功能, 可以得到空间的选择性. 环糊精 分子是 FDA 所认证的材料, 其来自于淀粉的生物降解, 所以对生物体无毒, 在自然环境中可以生物降解, 并且 对于光学无吸收和干涉, 因此可以应用于生物和光学材 料中. 更重要的是环糊精自组装化合物其分子结合力为 非键合的超分子作用, 因此可以具有刺激反应的性能, 能够构建具有刺激反应的智能材料, 在功能材料、传感 器和基因/药物载体等方面得到广泛应用.

聚轮烷的轮分子一个重要的方向就是胶体材料, 环 糊精可以沿着轴自由滑动, 因而有着滑轮作用. Ito 等 ${ }^{[26]}$ 首先报道了一种新的修饰的胶体材料, 以高分子 PEG 为骨架, 少量的环糊精分子加入后以二硝基苯封端形成 轮烷, 然后使用三氯三溙对高分子间的环糊精进行交 联, 形成了新型的超分子胶体材料. 这种材料不同于普 通的交联化的胶体, 作为交联功能团的轮烷结构可以自 由地沿着高分子链滑动, 称为滑轮效应, 因而对溶剂等 具有更好地适配性, 试验表明其对溶剂的吸收大大地增
强, 同时其黏弹性曲线也比相应地枝链化胶体延展性和 强度均有很大的强化作用. 最近他们 ${ }^{[27]}$ 扩展研究报道 了聚二甲基硅烷为骨架和 $\gamma$-CD 形成聚轮烷后(Scheme 21). 通过己二异氧酸酯结构交联得到了新型的有机物无机物杂化的聚硅烷的胶体.

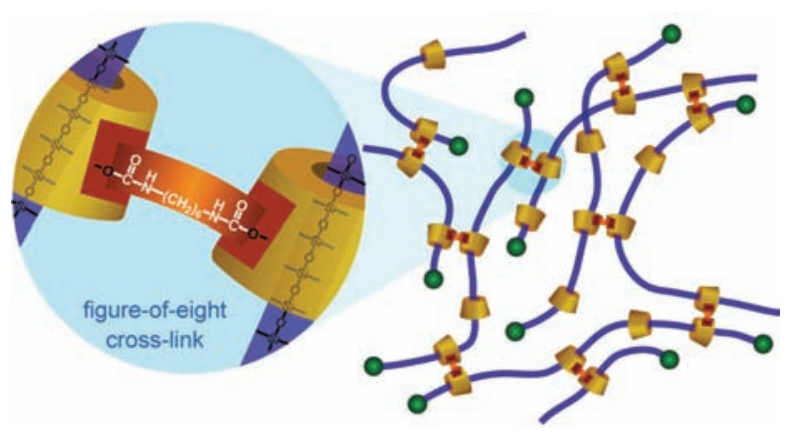

Scheme 21

Ito 等 ${ }^{[28]}$ 还应用聚轮烷结构作为骨架成功地得到了 聚轮烷液晶. 对氧基联苯通过已酸酯和聚轮烷骨架连接 得到了玻璃化点 $70{ }^{\circ} \mathrm{C}$ 和相转变点 $129{ }^{\circ} \mathrm{C}$ 的液晶 (Scheme 22).

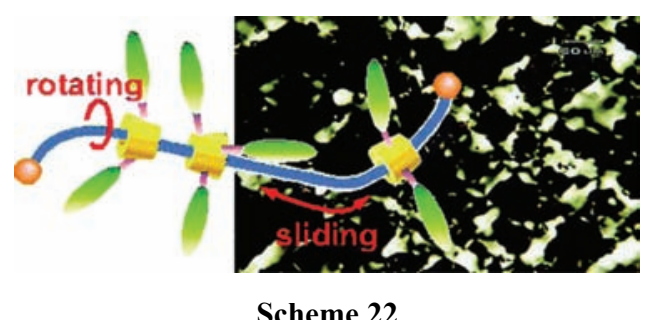

在凝胶方面，我们 ${ }^{[29]}$ 报道了多种不含有高分子骨 架的超分子凝胶结构, 其具有迅速地刺激响应性, 并且 避免了高分子难降解造成的环保问题. 三硝基苯桂皮酯 作为客体修饰 $\beta$-环糊精, 通过分子间主客体作用形成高 分子 I 结构, 环糊精间的氢键产生了交联作用, 得到了 水凝胶(Scheme 23). 这种水凝胶对多种刺激均具有相 应性,比如强客体分子、主体分子、加热以及尿素等. 通 过氨基取代环糊精的 6 位的美基，我们得到了一种酸碱 度和离子相应的有机凝胶, 通过 6 位氨基分子间的强氢 键作用, 环糊精自组装形成了高分子链状结构, 相缠绕 形成了有机凝胶 ${ }^{[30]}$. 酸碱度的改变和离子的添加可以 
减弱氨基分子间的氢键作用，而产生了溶胶化的改变. 这种超分子凝胶体系是一种高效的职能材料, 可以在药 物载体、分子器件等方面得到广泛应用.

利用轮烷的轮可以自由转动的特点, Park 等 ${ }^{[31]}$ 制备 了轮烷为高分子链交联体的胶体. 通过山葵过氧化酶和 过氧化氢反应, 得到以酪胺连接聚轮烷和壳聚糖结构的 水凝胶. 其研究了不同的反应条件不同结构的胶体的物 理性质, 尤其研究了结构对其自然降解性的影响. 此类 水凝胶对于生物体无毒, 有可能直接作为注射材料, 并 且通过结构控制其降解速度达到可控的药物释放.

Gao 等 ${ }^{[32]}$ 报道了一种两性的滑动超分子聚合物刷, 聚轮烷骨架修饰连接叠氮基团, 然后亲水性的 PEG 疏 水性的棕桐酸酯通过 click 反应同时修饰聚轮烷. 通过 调整 PEG 和棕榈酸酯的加入量, 可以得到适当修饰的 聚合物刷, 其在水和环己烷混合溶剂界面中, 自聚集形 成两性界面膜, 通过染料染色可以清晰观察到, 同时将
其旋涂在云母上, AFM 可以观测到其多空的薄膜结构 (Scheme 24). 该方法制备简单，可以有效地作为两性溶 剂的界面材料.

环糊精在高分子的聚合反应中也有着催化和选择 控制的作用. Zhang 等 ${ }^{[33]}$ 研究了二乙烯砜和已二胺在环 糊精作用下的聚砜一胺反应. 当单体的物质的量的比为 $1: 1$ 时, 得到了枝链化的聚合物结构, 其枝化度为 0.25 . 但是当环糊精分子的加入后，其枝化度得到明显的降 低，直到高浓度的环糊精作用下，可以完全得到直链状 的高分子结构. 当二乙烯砜和已二胺的物质的量的比为 $2: 1$ 时, 已二胺作用为化学交联剂而得到了多交联化 的胶体结构，同样添加环糊精分子，可又有效地抑制交 联化的结构, 得到枝链化的聚合物, 直到高浓度的环糊 精的存在下, 可以完全得到直链的聚砜胺结构(Scheme 25).
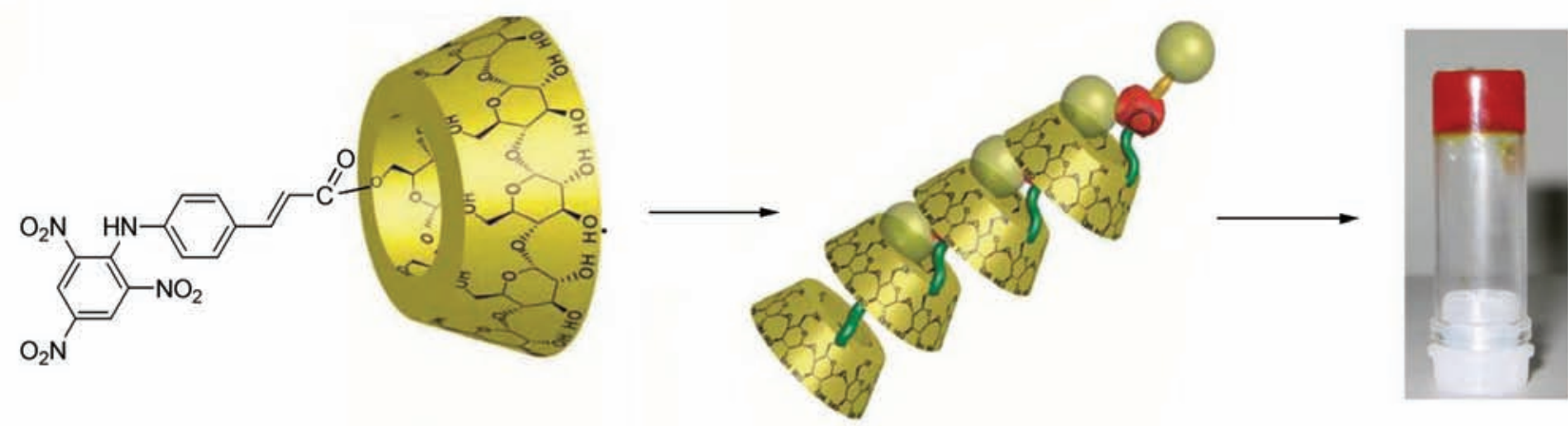

Scheme 23
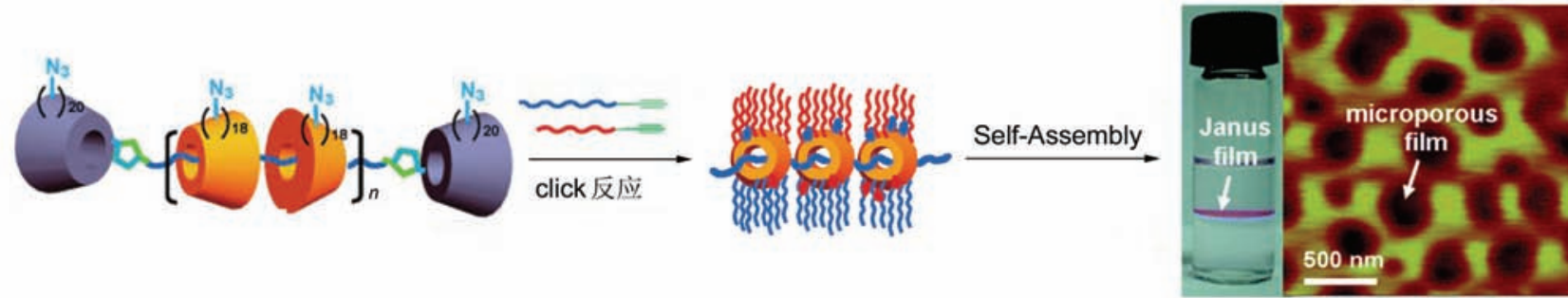

Scheme 24<smiles>C=CS(=O)(=O)C=C</smiles>

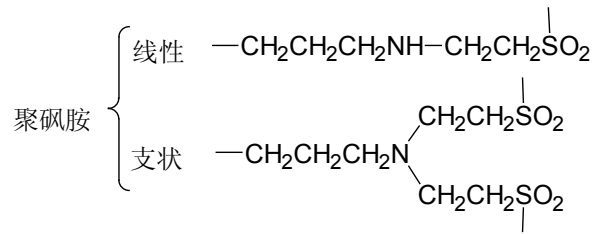

Scheme 25
Harada 等 ${ }^{[34 a]}$ 也利用环糊精对戊内酯等开环聚合反 应进行了研究, 发现环糊精的 2 位羟基基团对于开环聚 合在固相聚合有着催化效果，但是在少量的环糊精存在 下，由于开环的内酯堆积环糊精表面而抑制了开环聚合 的进一步进行，而在大量环糊精的作用下，开环的聚酯 进入到环糊精内腔中而释放了活性的 2 位羟基催化中 心, 让开环催化成功进行. 进一步研究发现, 环糊精的 催化作用是相邻的两个 2 差基基团的协同作用，因此利 用 2 位修饰的环糊精可以选择性地进行催化聚合, 比如 
其用顺式茴香酯修饰 2 位的 $\alpha$ 环糊精可以成功地催化成 内酯的开环催化, 而反式的茴香酯由于结构阻碍了邻位 的 2 基基团, 而不能催化开环聚合. 由于顺反式的茴香 酯结构可以在光的作用下, 选择性地相互转换, 从而进 行光控地催化聚合过程 ${ }^{[34 b]}$. 同时他们利用合成的环糊 精纳米球, 同样通过伪聚轮烷的机理, 成功地进行了高 分子的聚合催化反应 ${ }^{[33 c]}$. 环糊精催化剂高效、低毒、选 择性强, 不但具有表面活性剂的效果, 同样具有一定的 立体选择性.

在生物传感器上, 环糊精的超分子自组装同样也得 到了应用. Villalong 等 ${ }^{[35]}$ 设计制备了生物酶的双氧水电 极传感器. 金电极表面通过硫键修饰多环糊精的高分 子, 而后依次覆盖了多金刚烷的山葵过氧化酶和多环糊 精修饰的山葵过氧化酶, 从而形成了多层的过氧化酶修 饰的传感电极. 结果分析表明, 随着过氧化酶层数的增 加, 传感器的灵敏度也得到了很大的增强, 其中三层的 传感器结构灵敏度达到 $720 \mathrm{~A} /\left(\mathrm{mol} \cdot \mathrm{L}^{-1} \cdot \mathrm{cm}^{2}\right)$, 其最低检 测限度为 $2 \mu \mathrm{m}$, 同时保存 $30 \mathrm{~d}$ 以后性能仍旧有 $96 \%$, 其 性能远远好于未修饰的传感电极.

聚轮烷的轮分子在不同的温度下会可逆地穿入或 脱出高分子轴, 因此其可以设计温控的智能高分子材 料. Endo 等 ${ }^{[36]}$ 报道了热敏感的超分子轮烷系统, 其简单 地混合 $\alpha-\mathrm{CD}$ 和端氨基的 PEG 高分子. 在低温状态下, 高分子链插入到环糊精的空腔中, 形成伪轮烷结构, 其 状态为透明清澈的溶液. 而当升高温度时, 环糊精分子 滑出 PEG 的高分子链, 而使得伪轮烷结构破坏, 其状态 为混浊的胶体(Scheme 26). 这种热刺激反应相当敏感 并且可以多次重复.

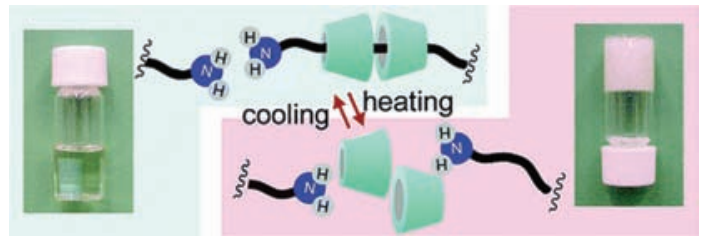

Scheme 26

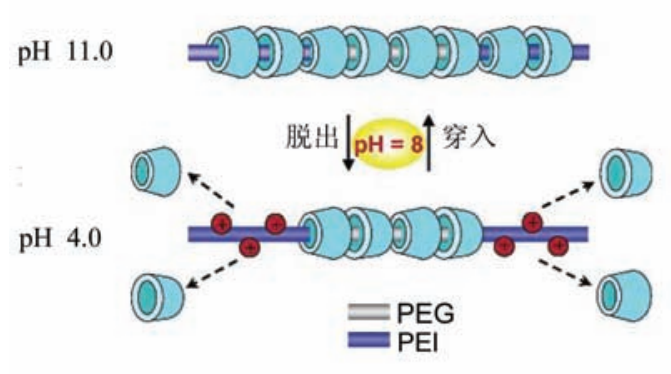

另一个常用的刺激源是 $\mathrm{pH}$ 值的变化. Yui 等 ${ }^{[37]}$ 报道 了一系列的 $\mathrm{pH}$ 敏感的伪聚轮烷, 使用不同分子量的线 性聚乙二胺(PEI)和环糊精反应, 在 $\mathrm{pH}$ 为 11.0 时得到了 最到产率达最高, 而在 $\mathrm{pH}$ 为 8.0 时, 由于氨基被正离子 化而没有轮烷生成. 因此，他们设计了许多 $\mathrm{pH}$ 敏感的 伪聚轮烷，以三段的 PEI-PEG-PEI 为高分子轴，以 $\mathrm{pH}$ 为 刺激源, 进行轮烷合成和脱落的控制(Scheme 27). 由于 细胞内外酸碱度环境不同, 因此 $\mathrm{pH}$ 敏感的材料在生物 材料范围内有着重要的潜在应用价值.

$\mathrm{Li}$ 等 ${ }^{[38]}$ 首先应用环糊精的轮烷在基因载体方面, 其以分段共聚的 PEG 和 PPG 为高分子轴, 与环糊精生 成超分子轮烷，而后以低分子聚乙二胺修饰环糊精形成 阳离子的轮烷作为基因载体. 他们对于该载体进行了系 统化的研究, 对于不同的肿瘤细胞在不同的条件下进行 研究，结果表明该结构的载体相对于传统的参比载体聚 乙二胺(PEI)具有毒性低, 基因转移率高的效果.

Harashima 等 ${ }^{[39]}$ 进一步研究了这类型的基因载体, 他们使用生物降解的连硫连接高分子轴和具塞分子. 这 种智能型的轮烷基因载体可以有效地和 $\mathrm{pDNA}$ 分子结 合，而使得 DNA 分子浓缩才聚成正电荷的纳米颗粒， 可以成功地进入细胞内, 在细胞内由于连硫键团被分解 而导致具塞分子的脱离, 从而环糊精分子从轴分子上脱 落，进行基因的靶向的智能释放(Scheme 28).

基于 PEI 轮烷的 $\mathrm{pH}$ 刺激反应性, Kim 等 ${ }^{[40]}$ 设计了 $\mathrm{pH}$ 敏感的超分子药物释放体系. 他们以多孔的硅土为 载体, 其多孔的空间可以吸附药物分子, 硅土表面修饰 连接大量的 PEI 分子, 在高 $\mathrm{pH}$ 的环糊精溶液中, 由于伪 轮烷的生成而阻止了小分子从空中的脱离, 形成了药物 载体. 当酸加入时, 环糊精分子从 PEI 分子上离去, 而 使得硅土表面的孔道被打开, 进行药物释放.

以多空硅土为载体, Feng 等 ${ }^{[41]}$ 报道了一种多刺激反 应的体系. 高分子链被 $\alpha-\mathrm{CD}$ 通过连硫键修饰, 然后其 连接到硅土表面. 药物分子存储于硅土的多孔空间内, 反式二偶氮分子通过和 $\alpha-\mathrm{CD}$ 的主客体作用成功封锁硅 土的表面空间, 形成药物载体. 这种可控药物释放体系

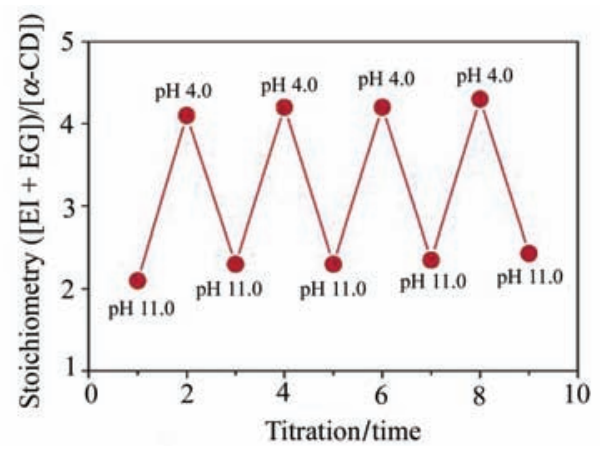

Scheme 27 


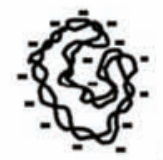

pDNA

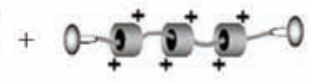

双硫键封瑞的正离子聚轮烷

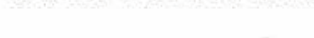
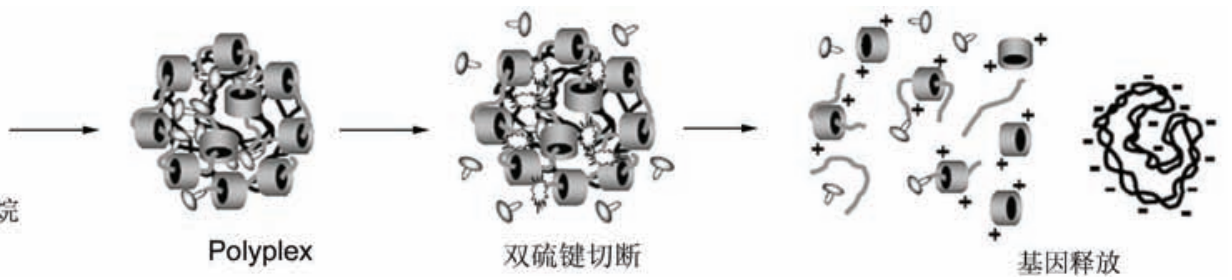

Scheme 28
在紫外光照下改变偶氮分子结构从反式到顺式而从环 糊精空腔中脱离, 具有交联作用的二偶氮分子的离去导 致药物分子的成功释放. 同样, 在添加竞争性的主体 $\beta-\mathrm{CD}$ 和连硫分子还原剂二硫苏糖醇(DTT)的作用下, 分 别由于偶氮分子被 $\beta-\mathrm{CD}$ 取代包合以及连硫分子被断开 而进行药物释放(Scheme 29). 这类智能药物释放体系 可以靶向地进行特定部位的药物释放治疗, 节省药物并 且不会对其他部位产生毒副效应, 是未来给药的趋势所 在.

Davis 等 ${ }^{[42]}$ 报道了首个应用环糊精超分子为基因载 体的临床试验, 其使用环糊精到聚合物和 siRNA 结合形 成基因纳米颗粒, 通过靶向作用治疗色素癌患者, 成功 地取得了临床基因治疗效果(Scheme 30). 这是首次超 分子聚合物作为基因载体的基因治疗的临床数据, 为未 来基因治疗提供了可能.

\section{4 结论和展望}

超分子的自组装结构由于其独有的特性越来越受 到化学家们的广泛关注, 尤其是环糊精相关的体系, 因 其源来自天然产物, 没有生物毒性, 且在光学没有相干 性, 在智能材料、生物医药和光学材料等方面都有潜在 的工业化应用. 通过数十年的基础研究, 环糊精的超分 子体系的技术路线和物理方法已经日益成熟, 环糊精的 基础研究已经渐趋完善, 当前的环糊精的超分子研究越 发朝着实际应用的方向发展. 化学家们努力应用环糊精 的超分子自组装体系构建智能材料, 未来在生物能源以 及信息方面都有着巨大的应用前景, 也是化学工作者的 努力方向所在, 环糊精相关的超分子体系仍将是未来超 分子的重要方向.

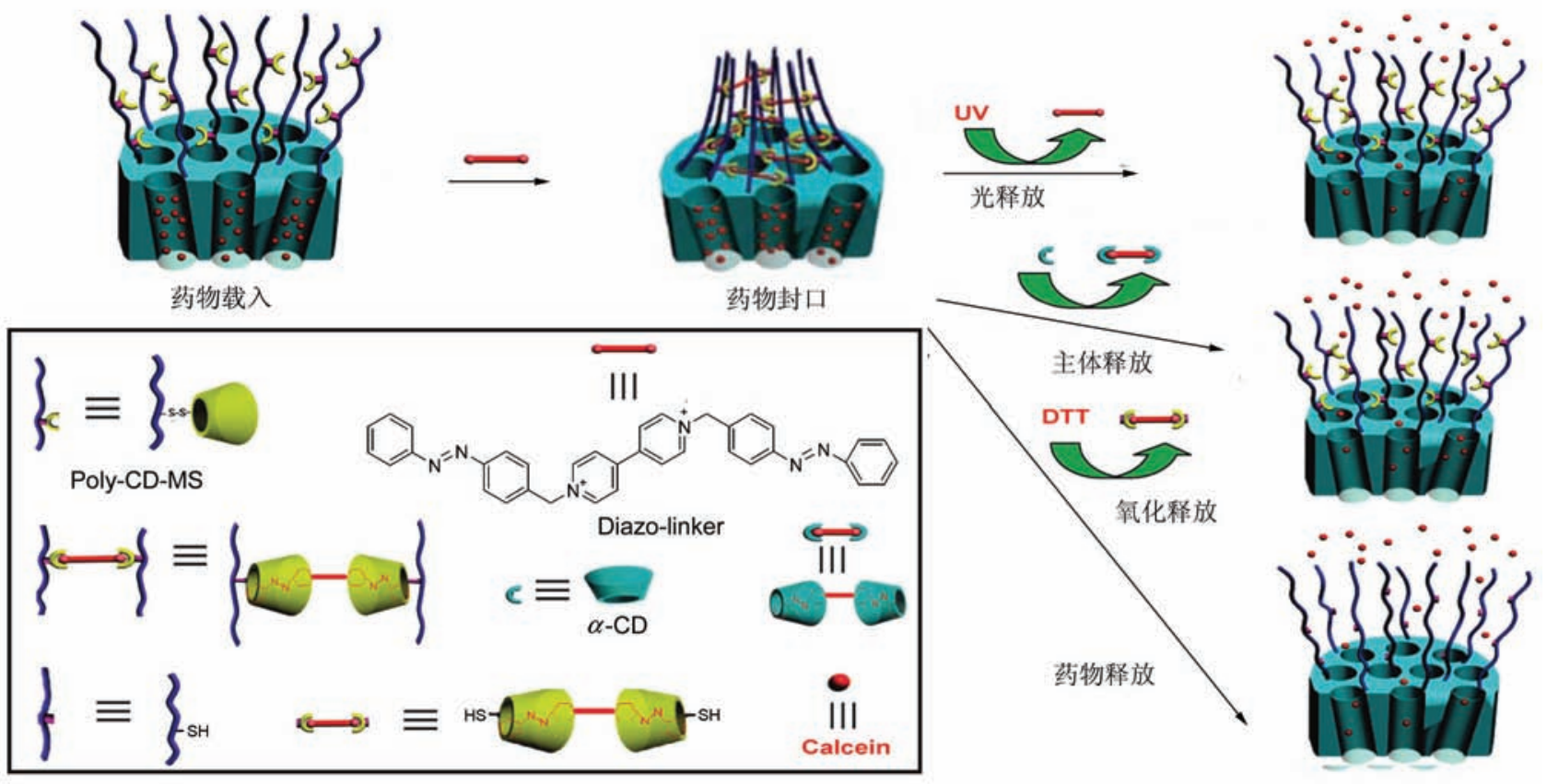

Scheme 29

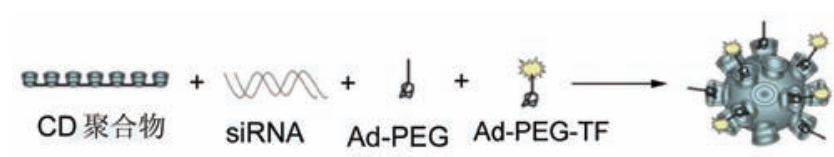

Scheme 30

\section{References}

[1] Liu, L.; Guo, Q.-X. J. Inclusion Phenom. Macrocyclic Chem. 2002, $42,1$.

[2] (a) Ogino, H. J. Am. Chem. Soc. 1981, 103, 1303. 
(b) Ogino, H.; Ohata, K. Inorg. Chem. 1984, 23, 2312.

[3] Hannak, R. B.; Farber, G.; Konrat, R.; Krauter, B. J. Am. Chem. Soc. 1997, 119, 2313.

[4] Rao, T. V. S.; Lawrence, D. S. J. Am. Chem. Soc. 1990, 112, 3614.

[5] Manka, J. S.; Lawrence. D. S. J. Am. Chem. Soc. 1990, 112, 2440.

[6] Liu, Y.; Zhao, Y.-L.; Zhang, H.-Y.; Li, X.-Y.; Liang, P.; Zhang, X.Z.; Xu, J.-J. Macromolecules 2004, 37, 6362.

[7] Nepal, D.; Samal, S.; Geckeler, K. E. Macromolecules 2003, 36, 3800 .

[8] (a) Born, M.; Ritter, H. Adv. Mater. 1996, 8, 149

(b) Yamaguchi, I.; Osakada, K.; Yamamoto, T. Macromolecules 1997, 30, 4288.

[9] Yamaguchi, I.; Osakada, K.; Yamamoto, T. Macromolecules 1997, $30,4288$.

[10] Dai, X.; Dong, C.; Fa, H.; Yan, D.; Wei, Y. Bismacromolecules 2006, 7, 3527.

[11] Liu, J.; Xu, R.; Kaifer, A. E. Langmuir 1998, 14, 7337.

[12] Harada, A.; Li, J.; Kamachi, M. Nature (London, U. K.) 1994, 370, 126.

[13] (a) Liu, Y.; Song, Y.; Wang, H.; Zhang, H.-Y.; Li, X.-Q. Macromolecules 2004, 37, 6370.

(b) Liu, Y.; Li, L.; Zhang, H. Y.; Zhao, Y. L.; Wu, X. Macromolecules 2002, 35, 9934.

[14] Guerrero-Martinez, A.; Avila, D.; Martinez-Casado, F.; Ripmeester, J.; Enright, G.; Cola, L.; Tardajos, G. J. Phys. Chem. B 2010, 114,11489 .

[15] Nakazono, K.; Takashima, T.; Arai, T.; Koyama, Y.; Takata, T. Macromolecules 2010, 43, 691.

[16] Araki, J.; Kagaya K.; Ohkawa, K. Biomacromolecules 2009, 10, 1947.

[17] Peres, B.; Richardeau, N.; Jarroux, N.; Guegan, P.; Auvray, L. Biomacromolecules 2008, 9, 2007.

[18] Dimitrius, M.; Terzis, A.; Coleman, A. W.; de Rango, C. Carbohydr. Res. 1996, 282, 125.

[19] (a) Amabilino, D. B.; Parsons, I. W.; Stoddart, J. F. Trends Polym. Sci. 1994, 2, 146.

(b) Raymo, F. M.; Stoddart, J. F. Trends Polym. Sci. 1996, 4, 208.

[20] Miyauchi, M.; Takashima, Y.; Yamaguchi, H.; Harada, A. J. Am. Chem. Soc. 2005, 127, 2984.

[21] Miyauchi, M.; Harada, A. J. Am. Chem. Soc. 2004, 126, 11418.

[22] (a) Miyawaki, A.; Takashima, Y.; Yamaguchi, H.; Harada, A. Chem. Lett. 2007, 36, 828.

(b) Miyawaki, A.; Takashima, Y.; Yamaguchi, H.; Harada, A. Tetrahedron 2008, 64, 8355

[23] Ohga, K.; Takashima, Y.; Takahashi, H.; Kawaguchi, Y.; Yamaguchi, H.; Harada, A. Macromolecules 2005, 38, 5897.

[24] Kuad, P.; Miyawaki, A.; Takashima, Y.; Yamaguchi, H.; Harada,

\section{A. J. Am. Chem. Soc. 2007, 129, 12630.}

[25] Galantini, L.; Jover, A.; Leggio, C.; Meijide, F.; Pavel, N.; Hugo, V.; Tellini, S.; Tato, J.; Tortolini, C. J. Phys. Chem. B 2008, 112, 8536.

[26] Okumura, Y.; Ito, K. Adv. Mater. 2001, 13, 485.

[27] Kato, K.; Inoue, K.; Kidowaki, M.; Ito, K. Macromolecules 2009, $42,7129$.

[28] Kidowaki, M.; Nakajima, T.; Araki, J.; Inomata, A.; Ishibashi, H.; Ito, K. Macromolecules 2007, 40, 6859.

[29] (a) Deng, W.; Yamaguchi, H.; Takashima, Y.; Harada, A. Chem. Asian J. 2008, 3, 687.

(b) Deng, W.; Yamaguchi, H.; Takashima, Y.; Harada, A. Angew. Chem., Int. Ed. 2007, 46, 5144.

[30] Deng, W.; Thompson, H. D. Soft Matter 2010, 6, 1884.

[31] Tran, N. Q.; Joung, Y. K.; Lih, E.; Park, K. M.; Park, K. D. Biomacromolecules 2010, 11, 617.

[32] Wu, J.; Gao, C. Macromolecules 2010, 43, 7139.

[33] Wan, H.; Chen, Y.; Chen, L.; Zhu, X.; Yan, D.; Li, B.; Liu, T.; Zhao, L.; Jiang, X.; Zhang, G. Macromolecules 2008, 41, 465.

[34] (a) Osaki, M.; Takashima, Y.; Yamaguchi, H.; Harada, A. J. Am. Chem. Soc. 2007, 129, 14452.

(b) Osaki, M.; Takashima, Y.; Yamaguchi, H.; Harada, A. Org. Biomol. Chem. 2009, 7, 1646.

(c) Osaki, M.; Takashima, Y.; Yamaguchi, H.; Harada, A. J. Org. Chem. 2009, 74, 1858.

[35] Camacho, C.; Matias, J.; Cao, R.; Matos, M.; Chico, B.; Hernandez, J.; Longo, M.; Sanroman, M.; Villalonga, R. Langmuir 2008, 24, 7654.

[36] Isobe, Y.; Sudo, A.; Endo, T. Macromolecules 2006, 39, 7783.

[37] (a) Lee, S. C.; Choi, H. S.; Ooya, T.; Yui, N. Macromolecules 2004, 37, 7464.

(b) Choi, H. S.; Yamamoto, K.; Ooya, T.; Yui, N. ChemPhysChem 2005, 6, 1081.

(c) Choi, H. S.; Hirasawa, A.; Ooya, T.; Kajihara, D.; Hohsaka, T.; Yui, N. ChemPhysChem 2006, 7, 1671.

(d) Joung, Y.-K.; Choi, H. S.; Ooya, T.; Yui, N. J. Inclusion Phenom. Macrocyclic Chem. 2007, 57, 323.

[38] Li, J.; Yang, C.; Li, H.; Wang, X.; Goh, S.; Ding, J.; Wang, D.; Leong, K. Adv. Mater. 2006, 18, 2969.

[39] Yamashita, A.; Kanda, D.; Katoono, R.; Yui, N.; Ooya, T.; Maruyama, A.; Akita, H.; Kogure, K.; Harashima, H. J. Controlled Release 2008, 131, 137.

[40] Park, C.; Oh, K.; Lee, S. C.; Kim, C. Angew. Chem., Int. Ed. 2007, $46,1455$.

[41] Liu, R.; Zhang Y.; Feng P. J. Am. Chem. Soc. 2009, 131, 15128.

[42] Davis, M.; Zuckerman, J.; Choi, C. H.; Seligson, D.; Tolcher, A.; Alabi, C.; Yen, Y.; Heidel, J.; Ribas, A. Nature 2010, 464, 1067.

(Qin, X.) 